\title{
EL REGISTRO DE Stegomastodon (MAMMALIA, GOMPHOTHERIIDAE) EN EL PLEISTOCENO SUPERIOR DE BRASIL
}

\author{
María Teresa ALBERDI ', José Luis PRADO ${ }^{2}$ \\ y Cástor CARTELLE ${ }^{3}$
}

1 Departamento de Paleobiología. Museo Nacional de Ciencias Naturales, CSIC. José
Gutiérrez Abascal, 2. 28006-Madrid (España). malberdi@ mncn.csic.es
2 INCUAPA- Departamento de Arqueología, Universidad Nacional del Centro UNC.
Del Valle 5737. B7400JWI -Olavarría (Argentina).
3 Universidad Federal de Minas Gerais. Belo Horizonte (Brasil). cartelle@csr.ufmg.br

Alberdi, M.T., Prado, J.L. y Cartelle, C. 2002. El registro de Stegomastodon (Mammalia, Gomphotheriidae) en el Pleistoceno superior de Brasil. [Stegomastodon (Mammalia, Gomphotheriidae) record from the late Pleistocene of Brazil.] Revista Española de Paleontología, 17(2), 217-235. ISSN 0213-6937.

\begin{abstract}
The most complete gomphothere skull from Pains (State of Minas Gerais) is described and compared with other mastodon remains from Brazil. Some of its characteristics are analysed and compared with those of other mastodons from different localities in South America, and is identified as Stegomastodon waringi. Also, the other remains from Toca dos Ossos (Bahia) and Bonito (Mato Grosso), are identified as Stegomastodon waringi. We consider that practically all Brazilian Gomphotheriidae must be included in only one subfamily (still unnamed), come from the late Pleistocene (Lujanian Land-mammal Age), and should all be assigned to Stegomastodon waringi. This species seems to have predominated in lower latitudes, and was better adapted to warm or temperate climatic conditions. Its migration routes from the north, towards the most austral sites are also discussed. Stegomastodon, a large gomphotherid, was dispersed through the east route and the coastal area, such as $S$. waringi from Santa Elena peninsula (Ecuador). Isotopic analysis of the latter species suggested an adaptive trend from mixed-feeder to grazer for the Pleistocene. Palaeoecological and palaeogeographic considerations of the South America mastodons are also given.
\end{abstract}

Keywords: Gomphotheriidae, Stegomastodon waringi, taxonomy, distribution, late Pleistocene, Brazil, South America.

\section{RESUMEN}

Se describe el cráneo más completo de gonfoterio procedente de Pains (Estado de Minas Gerais, Brasil) y se compara con otros restos de esta familia procedentes de otras localidades brasileñas. Se analizan algunos de sus caracteres más importantes y se compara con otros gonfoterios provenientes de distintas localidades de América del Sur. Se identifica como Stegomastodon waringi. Los restos de Toca dos Ossos (Bahia) y Bonito (Mato Grosso) también son identificados como Stegomastodon waringi. Nosotros también consideramos que todos los Gomphotheriidae brasileños deberían incluirse en una única subfamilia, sin nombre todavía, en un único género y una única especie: Stegomastodon waringi y proceden todos del Pleistoceno tardío. Esta especie parece estar mejor adaptada a vivir en latitudes bajas, y en condiciones climáticas cálidas o templadas. Se discuten sus posible vías de desplazamiento desde el norte hacia zonas o áreas más australes. Stegomastodon, gonfoterio de gran tamaño, se encuentra en localidades a lo largo de la ruta del este y en algunas áreas costeras, como es el caso de $S$. waringi de la península de Santa Elena (Ecuador). Los análisis isotópicos realizados sobre esta última especie sugieren una dieta mixta con cierta tendencia a pastadora. Se incluyen algunas consideraciones paleoecológicas y paleogeográficas de los gonfoterios de América del Sur.

Palabras clave: Gomphotheriidae, Stegomastodon waringi, taxonomía, distribución, Pleistoceno superior, Brasil, América del Sur. 


\section{INTRODUCCIÓN}

La familia Gomphotheriidae es un grupo ancestral del cual se originan otros grupos de proboscidios. Representantes de esta familia han tenido una radiación adaptativa dentro de Europa, Asia, y América del Norte desde el Eoceno tardío al Pleistoceno tardío.

Los gonfoterios sensu lato se registran en América del Norte desde el Mioceno medio hasta finales del Pleistoceno (Barstoviense final hasta el Rancholabreaniense). La mayor diversidad de gonfoterios se encuentra durante el Clarendoniense tardío hasta el Hemifiliense temprano. En este lapso se registran seis géneros: Gomphotherium, Rhynchotherium (en América Central), Amebelodon, Serbelodon, Platybelodon y Torynobelodon. Con posterioridad (en el Hemifiliense tardío) se reducen a tres géneros: Gomphotherium, Rhynchotherium y Amebelodon. Durante el Blanquiense, el registro es similar pero Rhynchotherium es el más abundante. Tanto Stegomastodon como Cuvieronius se registran desde el Blanquiense medio, mientras que los últimos registros del primero proceden del Irvingtoniense medio, el segundo desaparece en América del Norte a fines del Rancholabreaniense (Kurten y Anderson, 1980).

Los restos fósiles asignados a la familia Gomphotheriidae en América del Sur se registran desde el Pleistoceno medio hasta el Pleistoceno tardío. Esta familia se considera descendiente de los gonfoterios de América del Norte de donde pasaron a través de la vía Panameña a América del Sur, como parte del Gran Intercambio Biótico Americano (Webb, 1991). El registro más antiguo conocido son los mastodontes encontrados en la localidad boliviana de Tarija (1,0-0,7 Ma, MacFadden et al., 1983).

Casamiquela et al. (1996) han sugerido que los gonfoterios habrían ingresado en América del Sur durante el Plioceno o comienzos del Pleistoceno, pero no hay registros que confirmen esta hipótesis. Por su parte, Alberdi y Prado (1995; Prado et al., en prensa a) revisan la literatura sobre la mayor parte de los mastodontes de América del Sur, y plantean dos posibles vías migratorias: una por la Cordillera de los Andes que seguiría el género Cuvieronius y que podría llegar hasta Chile. La otra vía por las planicies brasileñas, que seguiría el género Stegomastodon y que llegaría a su nivel más austral en las Pampas de Argentina. Recientemente, Frassinetti y Alberdi (2000) revisan los mastodontes chilenos. Estos autores concluyen que a la vista de los datos existentes solo se puede confirmar la presencia de una especie de mastodonte en Chile: Cuvieronius hyodon. Prado et al. (en prensa a) han presentado una revisión, en parte bibliográfica, en la que resaltan la homogeneidad de las formas de América del Sur.

En Brasil, los primeros datos sobre registros de mastodontes datan de 1838, y corresponden a hallazgos realizados en la región de Lagoa Santa (Lund, 1839). Desde entonces se han sucedido una serie de hallazgos aislados que han sido sistematizados en gran parte en los trabajos monográficos de Simpson y Paula Couto (1955,
1957). Estos autores refieren los fósiles de gonfoterios procedentes de las regiones tropicales y templadas del sureste de Brasil a Haplomastodon waringi.

El objetivo principal de este trabajo es describir e identificar taxonómicamente los nuevos hallazgos de gonfoterios, principalmente los procedentes de Toca dos Ossos (Bahía) y el cráneo de Pains (Minas Gerais). Este estudio se complementa con una revisión de los materiales depositados en los principales museos de Brasil y la colección Lund en Dinamarca. Se analiza la distribución geográfica de los gonfoterios brasileños en función de las posibles vías migratorias en América del Sur, así como las condiciones de los ambientes en que vivieron.

\section{SITUACIÓN GEOGRÁFICA Y GEOLOGÍA}

Restos de mastodontes han sido registrados prácticamente en todo Brasil, desde la Amazonia (Acre y Pará) y la isla de Marajó en la desembocadura del río Amazonas hasta Río Grande do Sul. Los datos actuales no permiten identificar más de una especie. Ya en 1957 , Simpson y Paula Couto, en su revisión, plantearon que todos los restos conocidos hasta ese momento correspondían a una sola especie: Haplomastodon waringi.

Muchos de los hallazgos de la región intertropical extraamazónica ocurren en dos tipos principales de yacimientos: tanques y grutas. Los primeros consisten en depresiones naturales situadas en las rocas del basamento granítico, que se rellenan con sedimentos por la acción pluvial y que frecuentemente presentan un horizonte fosilífero. Los fósiles encontrados en este tipo de yacimientos y los de grutas calcáreas son sincrónicos (Cartelle, 1999), y se sitúan en el Pleistoceno final. El yacimiento de Araxá (Minas Gerais), estudiado por Simpson y Paula Couto (1957), es interpretado como fluvial y, por su composición específica absolutamente concordante con la de otros yacimientos, también se considera del Pleistoceno final. Se puede suponer que las modificaciones climáticas traducidas en la variación del régimen de lluvias fueron las que provocaron la acumulación de restos de animales en los tanques y grutas en un determinado período que coincidió con el final del Pleistoceno. En esos dos ambientes (tanques y grutas) no hay registros fosilíferos anteriores ni posteriores al final del Pleistoceno, lo que puede ser indicativo de la ocurrencia de un fenómeno aislado que provocó el acúmulo de restos que fosilizarían más tarde.

Los fósiles de Pains (Minas Gerais) fueron localizados, en los años 1998 a 2000, en la Gruta de Angá (20²5'35' S y $\left.45^{\circ} 37^{\prime} 52^{\prime \prime} \mathrm{O}\right)$, calcárea cuya entrada se localiza en el fondo de una fuerte pendiente de la dolina. Estos estaban depositados superficialmente, incrustados en una fina capa de arcilla y calcita, e hipermineralizados. A partir de la entrada, la gruta se bifurca y un riachuelo permanente corre en la galería del nivel más profundo. Ningún fósil se encontró en este tramo. Todos fueron recolectados en la galería situada en el nivel más alto o elevado. Además del cráneo, se encontró articulada la extremidad anterior 


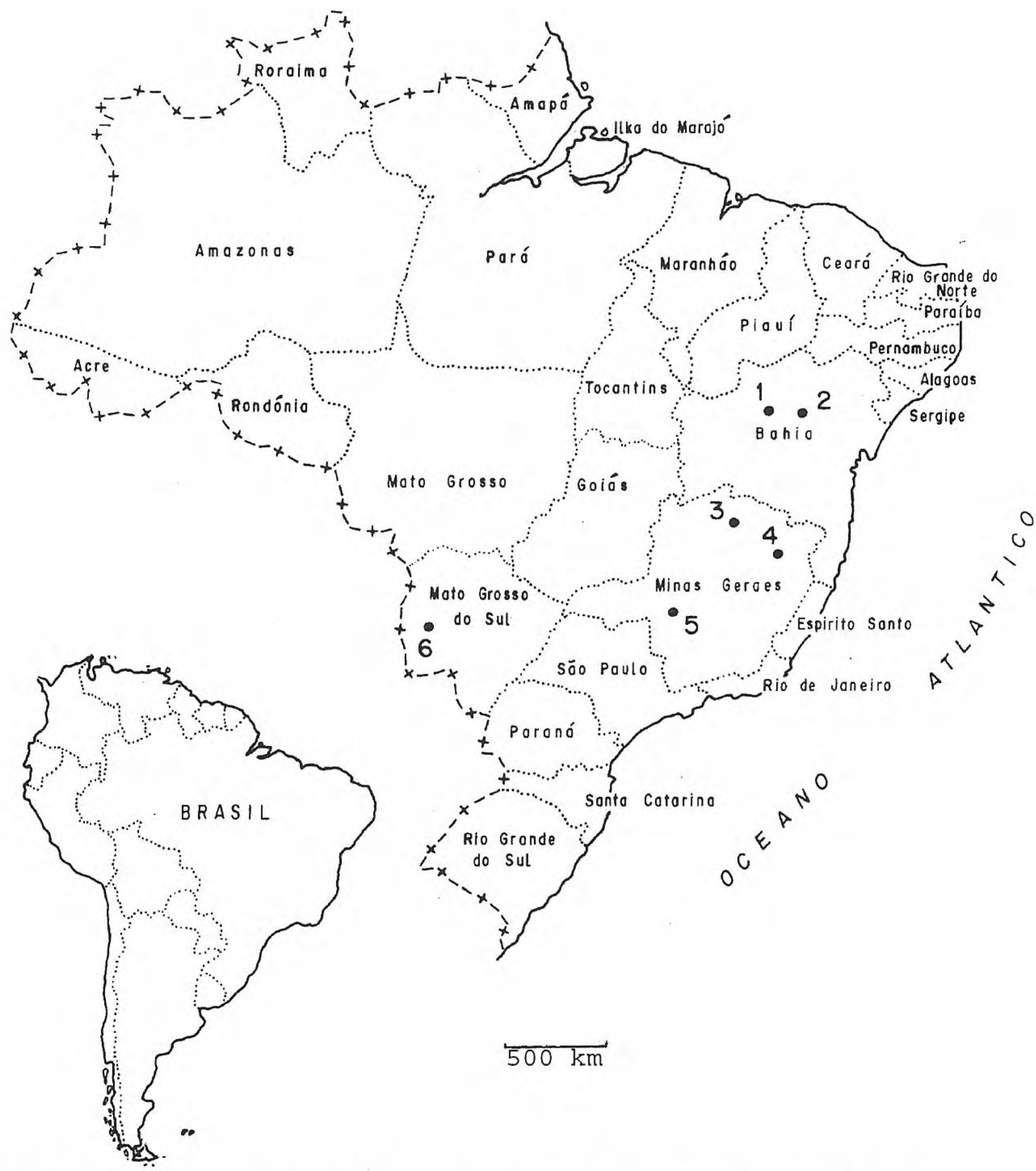

Figura 1. Distribución geográfica de los restos de mastodontes de las localidades brasileñas modificado de Simpson y Paula Couto (1957). Solo marcadas las localidades nuevas con relación al trabajo de Simpson y Paula Couto (1957). 1: Gruta dos Brejões (Morro do Chapeau); 2: Toca dos Ossos (Ourolândia); 3: Janauba; 4: Poté; 5: Gruta do Ingá (Pains); 6: Bonito.

Geographical location of the gomphoteres Brasilian localities modified from Simpson and Paula Couto (1957). Only marked the new localities: 1: Morro do Chapeau; 2: Ourolândia (Toca dos Ossos); 3: Janauba; 4: Poté; 5: Pains; 6: Bonito.

izquierda y el carpo. Probablemente, la inundación introdujo el animal y sólo se conservaron sus restos en la galería en la que la corriente del acuífero era menor o en donde las aguas formaron un pequeño lago endógeno de baja energía lo que propició que las piezas quedaran retenidas. Posteriormente, la acción del agua sobre las mismas fue poco intensa porque no presentan desgaste. Es este el único hallazgo brasileño de un cráneo muy completo con algunos de los huesos largos del mismo individuo, lo que nos permitirá calcular las proporciones del mismo.

Los restos de proboscidios procedentes de distintas localidades Brasileñas, muchos de los cuales fueron ya 
estudiados por Simpson y Paula Couto (1957), están representados en la Figura 1. En el Museu Nacional de Río de Janeiro (MNRJ) se encuentran depositados la mayor parte de los materiales descritos por estos autores. En este trabajo, ampliamos el registro con nuevas localidades que proceden de los municipios de Janaúba, Poté y Pains en el Estado de Minas Gerais, Jacobina y Morro do Chapéu en el Estado de Bahía, y Bonito en el Estado de Mato Grosso do Sul.

\section{MATERIAL Y MÉTODO}

Los restos considerados en este estudio están depositados en las colecciones del Museu Nacional de Rio de Janeiro (MNRJ), en el Museo de Historia Natural de la Universidad Federal de Minas Gerais en Belo Horizonte (MHN) y en las colecciones del Museo de la Pontificia Universidad Católica de Minas Gerais en Belo Horizonte (MCN), Brasil. También se incluyen los materiales depositados en la colección Lund del Museo Zoológico de Copenhague (ZMK), Dinamarca.

Los restos de gonfoterios fósiles de Brasil consisten en su gran mayoría en huesos y dientes aislados. Los restos de defensas también son escasos y en su mayoría carecen de procedencia exacta. Sólo algunos yacimientos han suministrado restos correspondientes al esqueleto apendicular, que por otra parte no presentan caracteres morfológicos que varíen entre ellos. Los restos más numerosos corresponden a dientes aislados, que si bien tienen una amplia variabilidad en talla, exhiben ciertos rasgos morfológicos que pueden apuntar algunas diferencias entre ellos.

Por ello, la presencia de un cráneo bastante completo de un ejemplar adulto procedente de la localidad de Pains (Minas Gerais), depositado en el MCN, es de gran importancia, así como el cráneo juvenil procedente de una gruta ubicada en el Municipio de Pedro Leopoldo, región de Lagoa Santa sensu lato, depositado en las colecciones del MHN, y la mandíbula expuesta en el MNRJ procedente de la localidad Pesqueira (hay un molde en el MCN). En el ZMK de Copenhague hay varios restos procedentes de Lapa da Escrivania (Lagoa Santa), entre ellos un cráneo juvenil, una defensa juvenil, y varios fragmentos de dientes.

Los restos depositados en el MCN son: Águas do Araxá: una mandíbula inferior derecha con M1, M2 y M3 en el alvéolo (figurado en Simpson y Paula Couto, 1957, Lám. 7); MCL-sn, un molde de mandíbula derecha con M3 muy gastado (figurado en Simpson y Paula Couto 1957, Lám. 5). Janaúba: MCL-3109, tres colina de un M3 o M2. Gruta dos Brejões (Bahía): MCL-3093, D4 inferior izquierdo y MCL-3087, fragmento de defensa. Ceará (sin localidad exacta): MCL-3070, hemimandíbula derecha con M2 muy desgastado y M3. Toca dos Ossos (Bahía): MCL3108, fragmento de mandíbula con restos de raíces; MCL3102-3101-3100, tres fragmentos de capas de defensas; MCL-3110, fragmentos de colina; MCL-3099, fragmento de diente; MCL-3101-3116, fragmentos de esmalte de diente; MCL-3091, P4 inferior izquierdo; MCL-3095, M1 superior derecho; MCL-3092, dos primeras colinas de un M3; MCL3096, M3 superior derecho; MCL-3097, M3 inferior derecho; MCL-3094, M3 inferior derecho; MCL-3092, dos fragmentos de colinas; MCL-3081 coneletes sueltos; MCL3083, fragmento de un M3; MCL-3085, fragmento de un D4 o M1; MCL-3078, fragmento germen de M2; MCL-3082, fragmento de D4 o M1; MCL-3088, talón de M3; MCL3079, fragmento de colina; MCL-3076, fragmento de una $1^{\text {a }}$ colina; MCL-3077, fragmento de diente; MCL-3080, fragmento de cono; MCL-3084 una colina; MCL-3074 fragmento de colina; MCL-3075, colina intermedia; MCL3071-3072-3073, fragmentos de colinas; MCL-3086, molar de leche; MCL-3089, dos colinas de un M1 o M2; sn, fragmentos de dientes de leche D3 o D4. Pains (Minas Gerais): cráneo sin número prácticamente entero, conserva la parte posterior izquierda y la anterior derecha, con M2 y M3 derechos e izquierdos, un húmero derecho, radio-ulna derecho y tibia de la misma localidad, y restos del carpo. Bonito (Mato Grosso do Sul): un M2 superior de leche, sin número.

Los restos depositados en el MHN son: Águas do Araxá: P32-MHN-40, M3 superior izquierdo. Poté (Minas Gerais), sin número M1 y M2 superior izquierdo. Municipio Pedro Leopoldo. Lapa Caetano: P2-MNH-33, un cráneo infantil con D2 y D3.

Los restos depositados en el MZK son: 1 cráneo juvenil, 1 defensa juvenil y varios fragmentos de dientes todos procedentes de las cuevas de la región de Lagoa Santa.

La mayor parte de estos restos están figurados en los trabajos de Simpson y Paula Couto (1957) y Cunha (1959). Nosotros figuraremos el cráneo y algunos restos de Toca dos Ossos que se describen aquí por primera vez.

Desde el punto de vista de la metodología, hemos seleccionado una serie de medidas y caracteres a partir de los establecidos por Tassy (1983), Boeuf (1983, 1992) sobre los restos de Anancus y Mammuthus de Chilhac, (Mazo 1977), y datos propios (MTA), con el fin de comparar y contrastar las diferencias y semejanzas entre los restos estudiados, en su mayoría correspondientes a la dentición, así como los escasos restos craneales y mandibulares. Los parámetros seleccionados para el cráneo se detallan en la figura 2: 1- longitud máxima del cráneo en el plano sagital; 2- distancia entre el vértex y el plano superior de los orificios nasales; 3- altura máxima de los orificios nasales; 4- longitud del premaxilar en el plano sagital; 5- anchura mínima entre las crestas temporales; 6- anchura máxima sub-orbitaria; 7- anchura de los premaxilares al nivel del orificio infraorbitario; 8- anchura del proceso nasal; 9anchura máxima del orificio nasal; 10- anchura entre los pseudomeatos auditivos externos; 11 - anchura entre los bordes externos de las fosas de articulación mandibulares; 12- anchura entre los bordes internos de las fosas de articulación mandibular; 13- anchura entre los bordes antero-interno de los molares; 14- anchura entre los bordes postero-interno de los molares; 15 - anchura de las coanas; 16- distancia entre el plano anterior de los molares y la espina palatina, plano sagital; 17- distancia entre la espina palatina y el borde anterior del foramen mágnum; 18distancia entre las apófisis pterigoideas del esfenoide y el borde anterior del foramen mágnum; 19- diámetro máximo antero-posterior de la fosa temporal a nivel de la arcada yugal; 20- distancia entre el punto más elevado de la cresta 
temporal y el borde antero-inferior del pseudomeato auditivo externo; 21 - distancia entre el punto ventral de la órbita y el borde antero-inferior del pseudomeato auditivo; 22- altura máxima de la órbita; 23 - distancia entre el punto más elevado de la cresta temporal y el borde alveolar; 24distancia entre el borde antero-inferior del premaxilar y la apófisis pterigoidea; 25 - distancia entre la punta del proceso zigomático del frontal y la apófisis pterigoidea; 26- distancia entre el vértex y la apófisis pterigoidea; 27-distancia entre el borde posterior de los cóndilos occipitales y la apófisis pterigoidea; 28- diámetro máximo antero-posterior de los premaxilares; 29- anchura máxima de la caja craneana; 30anchura de la fosa del ligamento nucal; 31 - anchura entre los bordes latero-externos de los cóndilos occipitales; 32 anchura entre los bordes latero-internos de los cóndilos occipitales; 33- altura máxima del cráneo (del vértex a la parte externo-inferior de los cóndilos occipitales; $34-$ distancia entre el vértex y la cima de la fosa del ligamento nucal; 35-longitud de la cresta de la fosa del ligamento nucal. Además hemos medido: DMO: diámetro máximo de la órbita; LC: longitud del cóndilo; DMFM: diámetro máximo del foramen mágnum; AMA: anchura del maxilar en el ápice; AVO: anchura ventral entre las órbitas; APV: anchura del premaxilar en su parte ventral; LSDMD: longitud serie dentaria maxilar derecha; LSDMI: longitud serie dentaria maxilar izquierda. Los parámetros y caracteres de la mandíbula seleccionados se detallan en la figura 3: 1proceso coronoide; 2 - incisura mandibular; 3- cóndilo articular; 4- fosa masetérica; 5- rama ascendente mandibular; 6- borde mandibular; 7- longitud de la serie dentaria; 8- forámenes mentonianos; 9- sínfisis mandibular; 10- longitud de la sínfisis en el plano sagital; 11 - fosa pterigoidea; a- longitud máxima de la mandíbula en el plano sagital; b- altura de la mandíbula a nivel anterior de la serie molar; c- altura de la misma a nivel posterior de la serie molar; d- anchura máxima de la rama ascendente; edistancia mínima entre el borde antero interno de los molares; a- ángulo que forma el borde lateral de la sínfisis o cresta interalveolar con la superficie oclusal de la dentición. En el caso de los dientes (Fig. 4) se han medido la longitud máxima y las anchuras máximas a nivel de cada una de las colinas (ver Tablas 1 y 2).

Debido a que los restos más numerosos son los dientes, hemos realizado un análisis estadístico bivariante con las dimensiones de los mismos (Fig. 5).

\section{SISTEMÁTICA PALEONTOLÓGICA}

Los primeros estudios sistemáticos sobre gonfoterios de América del Sur se deben a Cuvier (1806), quien analizó los restos encontrados por Dombey en Perú y Humboldt en Colombia, Ecuador (Imbabura), Bolivia (Chiquitos) y Chile (La Concepción). Cuvier agrupó estos "Mastodontes" con relación a su procedencia geográfica en "mastodonte des cordilleres" y "mastodonte humboldien", respectivamente. En 1920, Boule y Thevenin describieron los especímenes coleccionados en Bolivia como Mastodon andium. Asimismo, se produjo una gran controversia con
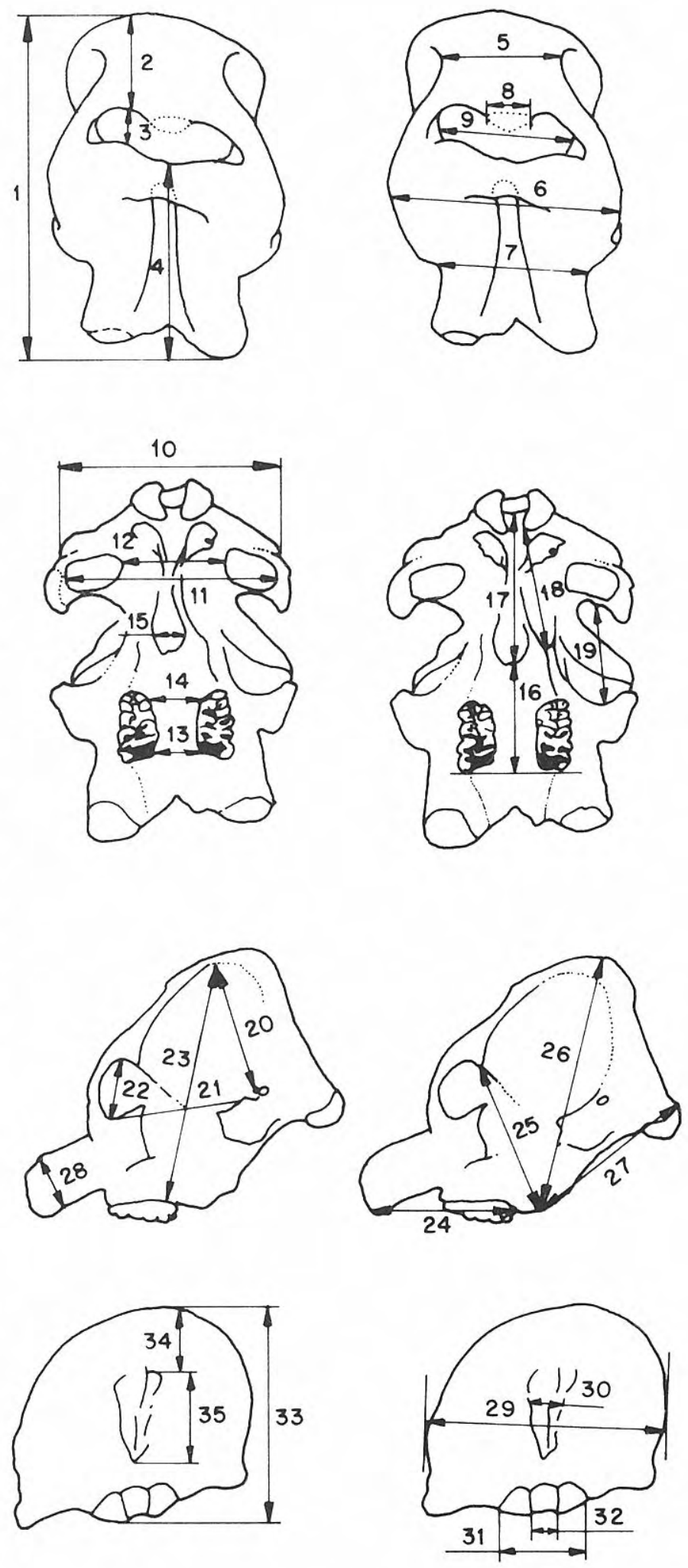

Figura 2. Nomenclatura y representación gráfica de las dimensiones del cráneo de mastodonte, modificado de Boeuf $(1983,1992)$. Explicación de los números 1 a 35 en el texto.

Nomenclature and graphic representation of the measurements of the gomphothere skull, modified from Boeuf $(1983,1992)$. Explanation of the numbers 1 to 35 in the text. 

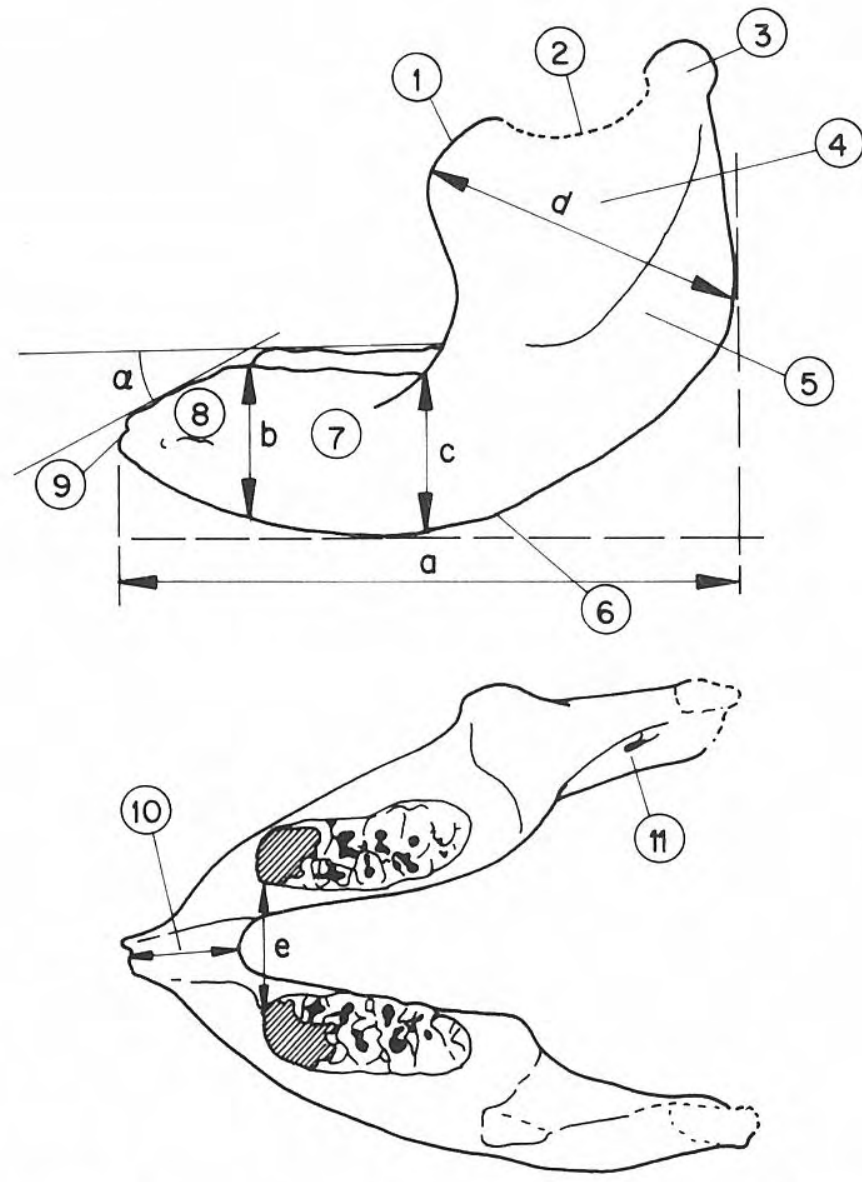

Figura 3. Representación gráfica de las dimensiones de la mandíbula de mastodonte, modificado de Boeuf (1983, 1992). Explicación de los números y las letras en el texto.

Nomenclature and graphic representation of the measurements of the gomphothere mandible, modified from Boeuf (1983, 1992). Explanation of the number and letters in the text.

respecto a la posición de estas especies en las clasificaciones propuestas para los proboscidios. Cabrera (1929: 74) considera que mastodontes y elefantes deben agruparse e incluye en la superfamilia Elephantoidea tres familias: Gomphotheriidae, Mammutidae y Elephantidae, en los cuales incluye a los gonfoterios bunodontos, las formas cigodontas, y los mastodontes estegodontos y elefantes, respectivamente. Este autor incluye las formas sudamericanas, que son bunodontas y brevirrostras, en la familia Gomphotheriidae. También crea la subfamilia Cuvieroniinae que él diferencia de Anancinae por la presencia de una banda de esmalte en los colmillos superiores. Hoffstetter (1952), como Cabrera, incluye todos los proboscidios de América del Sur en dos subfamilias: la subfamilia Cuvieroniinae como originaria de América del Norte y que se dispersó en América del Sur, en la cual incluye restos del norte de Ecuador Imbabura-, Colombia, Bolivia y Chile; y la subfamilia Anancinae, originaria de África y que se dispersó por Europa y parte de Asia, en la cual incluye parte de los

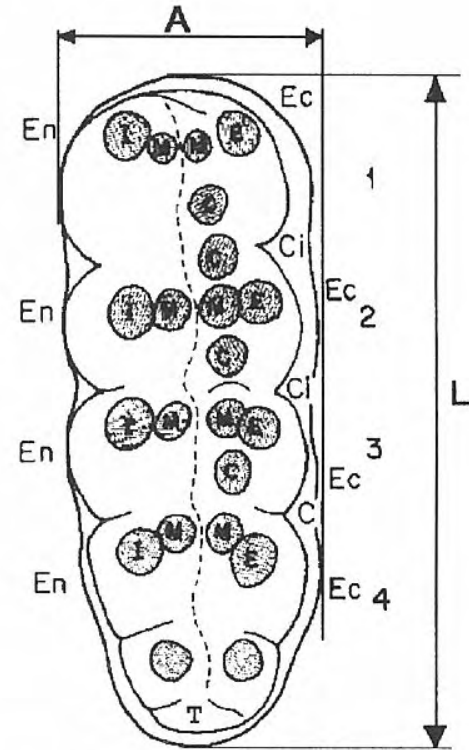

Figura 4. Representación gráfica de un esquema de un último molar, M3, de mastodonte bunodonto en cara oclusal. L: longitud máxima; A: anchura máxima a nivel de cada colina (números 1 a 4); En: entoconos o conos internos o linguales; Ec: ectoconos o conos externos o labiales; I: cono principal interno; $\mathrm{M}$ : mesoconos o conos próximos a la línea media; E: cono principal externo; $\mathrm{C}$ : conulos centrales; $\mathrm{Ci}$ : cíngulo. T: talón; Trazo discontinuo: línea media.

Schematic graphic of the gomphothere last molar, M3, in occlusal view. L: maximal length; A: maximal width at each loph (1, 2, 3, and 4); En: entocones (lingual main cusp of each loph); Ec: labial main cusp of each loph; I: the main cusp internal; M: mesocones or the cusp close to the median sulcus; $E$ : the main cusp external; $C$ : central conules; Ci: cingulum; T: talon; Dashed line: median sulcus.

restos de Ecuador y los materiales provenientes de Brasil y Argentina. Simpson y Paula Couto (1957) analizaron estos grupos en discusión (Anancinae y Cuvieroniinae) y observaron un patrón similar al de los elefantes modernos, donde se produce la pérdida de sus colmillos inferiores, el alargamiento de sus molares y el abombamiento del cráneo. Por consiguiente, ellos incluyeron todo los gonfoterios de América del Sur en un sola subfamilia: Anancinae, debido a su prioridad siguiendo las reglas de Nomenclatura Zoológica (Simpson, 1945; ICZN, 2000). También apuntan la posibilidad de olvidarse de las subfamilias e incluirlos directamente en la familia Gomphotheriidae. En una revisión reciente, Alberdi y Prado (1995; Prado et al., en prensa a) siguen el criterio propuesto por Simpson y Paula Couto (1957) dado que la morfología de las formas sudamericanas es muy homogénea. Recientemente, Tassy y Shoshani (1996: Apéndice B), proponen una clasificación de los Proboscidea donde incluyen los gonfoterios de América del Sur en la 


\begin{tabular}{ccccccccccc}
\hline sigla & tipo de diente & localidad & L & A 1 & A 2 & A 3 & A 4 & A 5 & T & A > \\
\hline R-2603-V & M3d & MNRJ & 186 & 76 & 73 & 72 & 71 & 65 &, & 76 \\
R-311-V & M3d & MNRJ & 210 & 80 & 88 & 92 & 82 & 63 &, & 92 \\
R-2140-V & M3d & MNRJ & 208 & 80 & 84,5 & 82,5 & 72 & 57 &, & 84,5 \\
R-2525-V & M3d & MNRJ & 203 & 85 & 90 & 89 & 79 & 57 &, & 90 \\
MCL-sn & M3d & MCN & 209 & 70 & 90 & 87 & 82 & 63 &, & 90 \\
MCL-3070 & M3d & MCN & 205 & 77 & 85,5 & 82 & 81,5 & 73 &, & 85,5 \\
MCL-3097 & M3d & MCN & 155 & 73 & 72 & 71 & 62 & 48 &, & 73 \\
MCL-3094 & M3d & MCN & 178 & 75 & 75 & 73,4 & 64 & 45 &, & 75 \\
MHN-sn & M3i & MHN & 195,5 & 74 & 72 & 75 & 73,2 & 62,5 &, & 75 \\
2492-V & M2i & MNRJ & 143,5 & 81 & 86,5 & 89 &, &, &, & 89 \\
2151-V & M2i & MNRJ & 122,5 & 66 & 74 & 73,2 &, &, &, & 74 \\
53-V54-V & M2d & MNRJ & 130 & 73 & 77 & 82 &, &, &, & 82 \\
53-V54-V & M2i & MNRJ & 134 & 79 & 81 & 91 &, &, &, & 91 \\
MCL-Sn & M2d & MCN & 133 & 72 & 74 & 84 &, &, &, & 84 \\
MCL-3070 & M2d & MCN & 114 & 67 & 74 & 79 &, &, &, & 79 \\
MCL-3107 & M2fi & MCN & 111 & 66 & 74 & 76 &, &, &, & 76 \\
MCL-3095 & M1d & MCN & 101,5 & 64 & 70 & 70,5 &, &, &, & 70,5 \\
55-V & M1d & MNRJ & 85 & 43 & 50 & 53 &, &, &, & 53 \\
309-V & M1i & MNRJ & 91 & 44 & 48 & 49 &, &, &, & 49 \\
SnPoté & M1i & MHN & 98,5 & 69 & 74 & 74 &, &, &, & 74 \\
MCL-3091 & D4i & MCN & 60 & 30 & 36 & 36 &, &, &, & 36 \\
MCL-3086 & D4d & MCN & 61,5 & 40,6 & 45,8 & 47 &, &, &, & 47 \\
MCL-Sn & D4i & MCN & 63 & 33,5 & 39,8 & 41,5 &, &, &, & 41,5 \\
MNH-33 & D3 & MHN & 54 & 37,5 & 36,7 & 36,5 &, &, &, & 37,5 \\
MNH-33 & D2 & MHN & 32 & 24 & 28,5 &, &, &, &, & 28,5 \\
ZMK-193 & D2 & ZMK & 25,5 & 17 & 20 &, &, &, &, &, \\
\hline
\end{tabular}

Tabla 1. Dimensiones, en milímetros, de los dientes inferiores de Stegomastodon waringi estudiados en este trabajo procedentes de Brasil. L: longitud máxima; A1: anchura de la primera colina; A2: anchura de la segunda colina; A3: anchura de la tercera colina; A4: anchura de la cuarta colina; A5: anchura de la quinta colina; T: talón; A >: la anchura mayor. MNRJ: Museu Nacional Rio de Janeiro; MCN: Museo de la Pontificia Universidad Católica de Minas Gerais; MHN: Museu de Ciências Naturais de la Universidad Federal de Minas Gerais; ZMK: Museo Zoológico de Copenhague, Dinamarca.

Measurements, in millimetres, of the Stegomastodon waringi lower teeth from Brazil. L: maximal length; A1: width of the first loph; A2: width of the second loph; A3: width of the third loph; A4: width of the fourth loph; A5: width of the fifth loph; T: talon; A>: the maximum width. MNRJ: Museu Nacional Rio de Janeiro; MCN: Museo de la Pontificia Universidad Católica de Minas Gerais; MHN: Museu de Ciências naturais de la Universidad Federal de Minas Gerais; ZMK: Zoologisk Museum of Copenhagen.

familia Gomphotheriidae Hay, 1922 y en la subfamilia Cuvieroniinae Cabrera, 1929. Tassy (1996) presenta un cladograma con dos grupos de gonfoterios: uno en el que incluye a los gonfoterios del Viejo Mundo, y otro en el que incluye las formas del Nuevo Mundo. Este autor (Tassy, 1996) considera que las formas americanas, en general, se relacionan más estrechamente con los gonfoterios tetralofodontos (cuatro colinas en los dientes intermedios y defensas inferiores reducidas) que con los trilofodontos (tres colinas y colmillos inferiores bien desarrollados y de sección más redondeada) de Eurasia. Este mismo autor apoya la posible relación filogenética entre Sinomastodon de China y las formas brevirrostrinas de América del Sur, que ya apuntaron Tobien et al. (1986, 1988). Por su parte, en el mismo libro, Shoshani (1996) incluye todos los gonfoterios de América del Sur en la subfamilia Cuvieroniinae sin ninguna justificación explícita. En contraposición, Chen (1999) cuestiona la relación entre Sinomastodon y los gonfoterios buno-brevirrostrinos-trilofondontos del Terciario superior de América del Norte. Este autor basa su discrepancia en que la morfología de los molares de Sinomastodon es más simple que la de los molares de gonfoterios norteamericanos, no existe registro en zonas intermedias entre China y Norte América, y que la aparición de las formas de gonfoterios en Norte América debería ser de la misma edad o algo más tardía que en China.

Nosotros consideramos, de acuerdo con Simpson y Paula Couto (1957), que no hay evidencias significativas que sustenten la inclusión de las formas de América del Sur en dos subfamilias diferentes. Tampoco creemos que la subfamilia Cuvieroniinae nominada por Cabrera pueda agrupar las distintas formas de gonfoterios encontradas 


\begin{tabular}{ccccccccccc}
\hline sigla & tipo diente & Institución & L & A 1 & A 2 & A 3 & A 4 & A 5 & T & A > \\
\hline Rio59-V & M3d & MNRJ & 190 & 81 & 79,8 & 78 & 69 & 53,5 &, & 81 \\
R-2602-V & M3d & MNRJ & 231 & 89 & 93 & 92 & 82 & 76 &, & 93 \\
R-2246-V & M3i & MNRJ & 202 & 97 & 100 & 99 & 95 & 73 &, & 100 \\
R-2950-V & M3i & MNRJ & 164 & 74 & 77 & 77 & 72 & 69 &, & 77 \\
cráneosn & M3d & MCN & 215 & 84 & 87 & 90 & 82 & 68 &, & 90 \\
cráneosn & M3i & MCN & 218 & 83 & 89,6 & 92 & 82,5 & 67 &, & 92 \\
MHN-40 & M3i & MHN & 195,6 & 95,2 & 91,2 & 85 & 77 & 61 &, & 95,2 \\
cráneosn & M2d & MCN & 125 & 80 & 81 & 76 &, &, &, & 81 \\
cráneosn & M2i & MCN & 132 & 79 & 85,3 & 82,8 &, &, &, & 85,3 \\
Poté s/n & M2i & MHN & 125,3 & 86,7 & 89 & 88,5 &, &, &, & 89 \\
2490-V & M1d & MNRJ & 80 & 55 & 61 & 58 &, &, &, & 61 \\
MCL-3095 & M1d & MCN & 101,5 & 64 & 70 & 70,5 &, &, &, & 70,5 \\
SnPoté & M1i & MHN & 98,5 & 69 & 74 & 74 &, &, &, & 74 \\
MCL-3086 & D4d & MCN & 61,5 & 40,6 & 45,8 & 47 &, &, &, & 47 \\
MCL-3080 & ?D3 & MCN & 62 & 42 & 39 & 35 & & & & 42 \\
SnBonito & ?D3 & MCN & 59 & 40 & 38 & 37 & & & 40 \\
MZK-Lund & D3i & MZK & 54,5 & 36 & 42 & 40,5 &, &, &, & 42 \\
MZK-Lund & D3d & MZK & 51,5 & 36 & 42 & 39 &, &, &, & 42 \\
MHN-33 & D3 & MHN & 54 & 37,5 & 36,7 & 36,5 &, &, &, & 37,5 \\
ZMK-Lund & D2i & MZK & 33,5 & 24,5 & 30,5 &, &, &, &, & 30,5 \\
ZMK-Lund & D2d & MZK & 34 & 24 & 31 &, &, &, &, & 30,5 \\
ZMK-193 & D2 & MZK & 25,5 & 17 & 20 &, &, &, &, & 20 \\
MHN-33 & D2 & MHN & 32 & 24 & 28,5 &, &, &, &, & 28,5 \\
\hline
\end{tabular}

Tabla 2. Dimensiones, en milímetros, de los dientes superiores de Stegomastodon waringi estudiados en este trabajo procedentes de Brasil. Abreviaturas como en Tabla 1.

Measurements, in millimetres, of the Stegomastodon waringi upper teeth from Brazil. Abbreviations as in Table 1.

en América del Sur, por ser demasiado especifica para el género Cuvieronius. Por otra parte, los recientes trabajos de Lambert (1996) y Dudley (1996) indican claramente que el género Anancus nunca llegó a Norte América, lo que obviamente dificulta la inclusión de los gonfoterios de América del Sur en la subfamilia Anancinae. Hasta tanto no se realice una revisión filogenética (en curso de realización), consideramos oportuno incluir estos géneros en la familia Gomphotheriidae.

Los gonfoterios de América del Sur presentan un modelo generalizado para la mayoría de sus representantes. Están caracterizados por la presencia de un cráneo braquicéfalo con una tendencia a alcanzar una forma elefantoidea y una mandíbula de tipo brevirrostra. Las defensas superiores varían de más o menos alargadas, más o menos curvas, e incluso con más o menos torsión. Con o sin banda de esmalte, que muchas veces desaparece en los especímenes adultos. Los molares bunodontos y braquidontos o subhipsodontos suelen presentar una disposición angular de los conos internos y externos en las últimas colinas, con figuras treboladas en la superficie oclusal, más o menos complicadas, producidas por el desgaste. Los M2 son trilofodontos con un talón desarrollado. El M3 varía desde tetralofodonto a heptalofodonto.

Familia Gomphotheriidae Cabrera, 1929 Género Stegomastodon Pohlig, 1912
1842 Mastodon; Lesson, 157.

1906 Mastodon; Winge, 48.

1928 Bunolophodon; Spillmann, 67.

1929 Notiomastodon Cabrera, 90-96, figs. 2 y 4.

1950 Stegomastodon (Haplomastodon) Hoffstetter, 22, figs. 2 y 3.

1952 Haplomastodon (Aleamastodon) Hoffstetter, (en parte), 208.

1957 Haplomastodon Hoffstetter; Simpson y Paula Couto, 166.

Para más detalles ver Cabrera (1929) y Osborn (1936), entre otros.

Especie tipo: Mastodon mirificus Leidy, 1858.

\section{Distribución estratigráfica}

El género se registra desde el Plioceno tardío hasta el Pleistoceno temprano en las regiones centrales y occidentales de América del Norte. En América del Sur está presente en el Pleistoceno medio y tardío de Brasil, Argentina, Paraguay y Uruguay, y el Pleistoceno tardío de Ecuador, Colombia y Venezuela.

\section{Distribución geográfica}

El género se registra en América del Sur desde el sitio de Taima-Taima en Venezuela (Bryan et al., 1978; Bryan, 1986; Casamiquela et al., 1996), y la península de Santa Elena en Ecuador (Hoffstetter, 1952) hasta las pampas de 


\begin{tabular}{|c|c|c|c|c|c|c|c|c|c|c|c|c|c|c|c|}
\hline medidas & 1 & 2 & 3 & 4 & 5 & 6 & 7 & 8 & 9 & 10 & 11 & 12 & 13 & 14 & 15 \\
\hline Pains & 980 & 304 & 163 & 580 & 207 & 520 & 448 & 112 & 214 & 660 & 540 & 200 & 140 & 102 & 79 \\
\hline MHN-33 & & & & 243 & & 197 & 138,5 & & & & & & 74 & 64 & \\
\hline ZMK & 290 & 100 & 46 & 163 & 144 & 194 & 121 & 46 & 69 & & & 126 & 74 & 49 & 43 \\
\hline
\end{tabular}

\begin{tabular}{|c|c|c|c|c|c|c|c|c|c|c|c|c|c|c|}
\hline medidas & 16 & 17 & 18 & 19 & 20 & 21 & 22 & 23 & 24 & 25 & 26 & 27 & 28 & 29 \\
\hline Pains & 229 & 326 & 246 & 293 & 445 & 402 & 217 & 625 & 690 & 476 & 650 & 395 & 216 & 775 \\
\hline MHN-33 & 119 & & & $102 ; 98$ & & 159 & 45,$6 ; 62,3$ & & $127 ; 122$ & 130 & & & 54,$5 ; 51,5$ & \\
\hline ZMK & 174 & & & $79 ; 78$ & & & 98 & 221 & 200 & 150 & 212 & & 43 & \\
\hline
\end{tabular}

\begin{tabular}{|c|c|c|c|c|c|c|c|c|c|c|c|c|c|c|}
\hline medidas & 30 & 31 & 32 & 33 & 34 & 35 & DMO & LC & LOO & AMA & AVO & APV & LSDMD & LSDMI \\
\hline Pains & 83 & 227 & 80 & 495 & 126 & 130 & 190 & 227 & 80 & & & & & \\
\hline MHN-33 & & & & & & & & & & & & & 88 & 89 \\
\hline ZMK & & & & & & & & & & 140 & 214 & 130,5 & & \\
\hline
\end{tabular}

Tabla 3. Dimensiones, en milímetros, de los cráneos de Stegomastodon waringi estudiados en este trabajo procedentes de Brasil. Los números representan los distintos parámetros y caracteres explicados en el texto. Pains (MG): cráneo casi completo objeto principal de este estudio, depositado en el MCN; MHN-33: Museu de Ciências Naturais de Belo Horizonte; ZMK: Zoologisk Museum de Copenhague.

Measurements, in millimetres, of the Stegomastodon waringi skull from Brazil. The numbers represent the parameters and characters explain in the text. Pains $(M G)$ : the almost complete skull main object of this study, stored at the MCN; MHN-33: Museu de Ciências Naturais de Belo Horizonte; ZMK: Zoologisk Museum of Copenhagen.

Argentina y Uruguay (Cabrera, 1929; Simpson y Paula Couto, 1957; Mones y Francis, 1973); y en Brasil y posiblemente en Paraguay siguiendo la ruta de dispersión oriental (Proaño, 1922; Hoffstetter, 1952; Simpson y Paula Couto, 1955, 1957).

\section{Diagnosis}

Sintetizada a partir de Cabrera (1929) y Simpson y Paula Couto (1957). Gomphotheriidae con cráneo corto y alto, tipo elefantoideo, menos deprimido que Cuvieronius. Mandíbula brevirrostra con dientes intermedios bunodontos trilofodontos y con talón fuerte (D3, D4, M1, M2). M3 con moderada alternancia de los conos externos e internos sobre las colinas o lofos posteriores, moderadamente oblicuos. Patrón de figuras de desgaste en doble trébol de simple a complicado. Las defensas varían de curvadas a casi rectas, sin esmalte en general en adultos; sobre algunos individuos juveniles se observa banda de esmalte.

\section{Stegomastodon waringi (Holland, 1920)}

1842 Mastodon brasiliensis Luna; Lesson, 157, nomen nudum.

1906 Mastodon andium Cuvier; Winge, 48, nec Cuvier, 1806, 413.

1920 Mastodon humboldti Cuvier, 1806; Boule y Thevenin, 68.

1920 Mastodon waringi Holland, 229.

1922 Mastodon chimborazi Proaño, 13.

1928 Bunolophodon Ayora Spillmann, 67.

1931 Bunolophodon postremus Spillmann, 73.

1948 Notiomastodon vidali Castellanos, 139.

1952 Haplomastodon (Aleamastodon) guayasensis Hoffstetter, 208.
1952 Especies B de La Carolina, Hoffstetter, 224.

1952 Stegomastodon brasiliensis Hoffstetter, 222.

1957 Haplomastodon waringi (Holland, 1920); Simpson y Paula Couto, 171

1995 Haplomastodon chimborazi (Proaño, 1922); Ficcarelli et al., 753-754.

1996 Haplomastodon waringi (Holland, 1920); Casamiquela et al., 316.

\section{Holotipo}

CM-11033 descrito por Holland (1920:229) como una mandíbula incompleta y otros fragmentos procedentes de Pedra Vermelha, Bahía, Brasil. Simpson y Paula Couto (1957: 172) mencionan que en la colección del Carnegie Museum sólo hay tres fragmentos de molares, la punta de un colmillo, un fragmento de dentina del colmillo, y parte del extremo distal de una tibia, bajo el número CM-11033.

\section{Distribución estratigráfica}

Stegomastodon waringi es característica del Pleistoceno medio y tardío.

\section{Distribución geográfica}

Se registra en Ecuador, en la península de Santa Elena, en Taima-Taima en Venezuela, en Lagoa Santa en Minas Gerais y Bonito en Mato Grosso do Sul, y posiblemente en Río Grande do Sul (ver Simpson y Paula Couto, 1957), en Brasil.

\section{Diagnosis}

El cráneo menos elefantoideo que en la especie tipo y menos deprimido que en Cuvieronius. La superficie oclusal tiene un patrón de desgaste en trébol relativamente complicado. Con una tendencia al desplazamiento de los conos internos frente a los externos mayor que en Cuvieronius. Las defensas con una 
curvatura simple o casi rectas, o con una torsión muy ligera, con banda de esmalte en algunos individuos juveniles y normalmente sin esmalte en adultos.

\section{Discusión}

Nosotros entendemos que la especie " $H$. waringi" debe emplazarse en el género Stegomastodon y no en el género Haplomastodon ya que el primero tiene prioridad. En nuestra opinión, no hay, por el momento, argumentos de peso para pensar que Stegomastodon no pasó a América del Sur a través del Istmo de Panamá. Simpson y Paula Couto (1957: 185) consideran que Haplomastodon no está bien definido, y de hecho fue descrito como un subgénero de Stegomastodon (ver Hoffstetter, 1950:24 y siguientes). En general las diferencias entre las dos especies de Stegomastodon citadas por Alberdi y Prado (1995) y Prado et al. (en prensa a, b) son sutiles y seguramente estén más relacionadas, en su caso, con diferencias que pudieran indicar condiciones ambientales algo diferentes. En la actualidad estamos llevando a cabo un estudio detallado de todos los mastodontes sudamericanos con el fin de dilucidar y profundizar en las diferencias y semejanzas entre ellos.

\section{DESCRIPCIÓN DEL MATERIAL DE BRASIL}

Entre los restos de mastodontes de Brasil destaca el cráneo bastante completo procedente de Pains (Minas Gerais). Este cráneo, sin número, carece de defensas y conserva la parte anterior del maxilar izquierdo y en la parte posterior conserva el lado derecho de la bóveda craneal (Figs. 6, 7a-7g). En el mismo, se observan perfectamente los alvéolos de las defensas ligeramente divergentes, así como la parte anterior de la sínfisis bastante recta en comparación con la de los cráneos conocidos de Cuvieronius hyodon de Tarija. Uno de estos está expuesto en el Museo Argentino de Ciencias Naturales "Bernardino Rivadavia", y tiene las defensas divergentes, dirigidas hacia fuera y con fuerte torsión; otro, está expuesto en el Museum National d'Histoire Naturelle de Paris, y tiene las defensas menos divergentes y dirigidas hacia dentro, también con fuerte torsión.

El premaxilar del cráneo de Pains presenta un surco, hendidura sagital, poco divergente en su extremo mesial. Ventralmente, en el maxilar, conserva M2 y M3 derechos e izquierdos, y se observa la huella del alvéolo de M1 en el lado izquierdo. M2 derecho está bastante deteriorado. Los M2 bastante desgastados, con tres colinas y un talón fuerte, mientras que los M3 presentan 5 colinas y un talón robusto, con línea media marcada y cónulos centrales fuertes, mayoritariamente internos -algunos externos-, a la línea media, con el desgaste iniciado sólo en las primeras colinas. Figuras treboladas características, aunque algo más complicadas ya que presentan cónulos centrales que van a formar parte también de las figuras más externas. Los conos externos presentan división ternaria. Los conos externos frente a los internos presentan una moderada oblicuidad, formando a modo de un ligero ángulo que comienza a partir de la segunda colina de M3, y se va cerrando progresivamente desde ésta hasta la $5^{\text {a }}$ colina $\left(150^{\circ}\right.$ en la segunda colina, hasta $115^{\circ}$ en la quinta). En algunos casos, esto lleva consigo un cierto desplazamiento de los conos externos frente a los internos, presentando una ligera anancoidía, que podemos observar sobre la $5^{\mathrm{a}}$ colina. Este diente presenta cíngulo mesial fuerte prolongándose lingualmente hasta el segundo valle. La bóveda craneana está ligeramente abombada, pero este abombamiento no es tan acusado como apunta Cabrera (1929), ni tiene una acusada diferencia con los cráneos de Cuvieronius procedentes de Tarija. La parte posterior o nucal está rota en el lado izquierdo, conservando la otra parte de la bóveda, donde se observa claramente y bien desarrollada la fosa nucal y el ligamento nucal. Las dimensiones, en milímetros, de los parámetros tomados sobre el cráneo de Pains (Minas Gerais, Brasil) están reseñadas en la Tabla 3, y la referencia a los mismos representada en la Figura 2.

Los restos dentarios de este cráneo se sitúan aproximadamente entre los más grande de los dientes de América del Sur, según se puede observar en los diagramas bivariantes de M2 y M3 (Tabla 2; Fig. 5).

Los restos del esqueleto apendicular que corresponden al mismo animal que el cráneo se reducen a un húmero, un radio, una ulna, una tibia, y los siguientes restos del carpo izquierdo: ulnar o pisiforme, intermedio o lunatum, carpiano II o trapezoide, carpiano III o magno, y unciforme. Sus dimensiones en milímetros son:

- Húmero: longitud máxima 795/anchura distal máxima 265 (latero-medial)/anchura proximal máxima 300 (lateromedial);

- Radio: longitud máxima: 610/anchura distal máxima: 167/anchura proximal máxima: 117;

- Ulna: longitud máxima: 691/anchura distal máxima: 183 (latero-medial)/anchura proximal máxima 223 (lateromedial)/anchura antero-posterior en el olécranon: 335;

- Tibia. longitud máxima 600/ anchura proximal máxima: 231(latero-medial)/anchura distal máxima: 182.

Del carpo izquierdo:

- ulnar o pisiforme: anchura máxima (latero-medial): 163/espesor máximo (antero-posterior): 124/longitud máxima (próximo-distal): 100;

- intermedio o lunatum: anchura máxima (latero-medial): 101/espesor máximo (antero-posterior): 133/longitud máxima (próximo-distal): 75;

- II carpal o trapezoide: anchura máxima (latero-medial): 71/espesor máximo (antero-posterior): 100/longitud máxima (próximo-distal): 63;

- III carpal o mágnum: anchura máxima (latero-medial): 100/espesor máximo (antero-posterior): 105/longitud máxima (próximo-distal): 92;

- IV-V carpal o unciforme: anchura máxima (lateromedial): 126/espesor máximo (antero-posterior): 122/longitud máxima (próximo-distal) 97.

Aunque no disponemos de todos los restos del esqueleto apendicular proximal, podemos apuntar una altura aproximada en cruz del animal que portaba el cráneo de Pains en torno a unos 2,5 metros.

El cráneo juvenil (MHN-33), procedente de la región de Lagoa Santa sensu lato, conserva las series dentarias, donde están enteros D2 y D3, mientras que D4 inicia su 


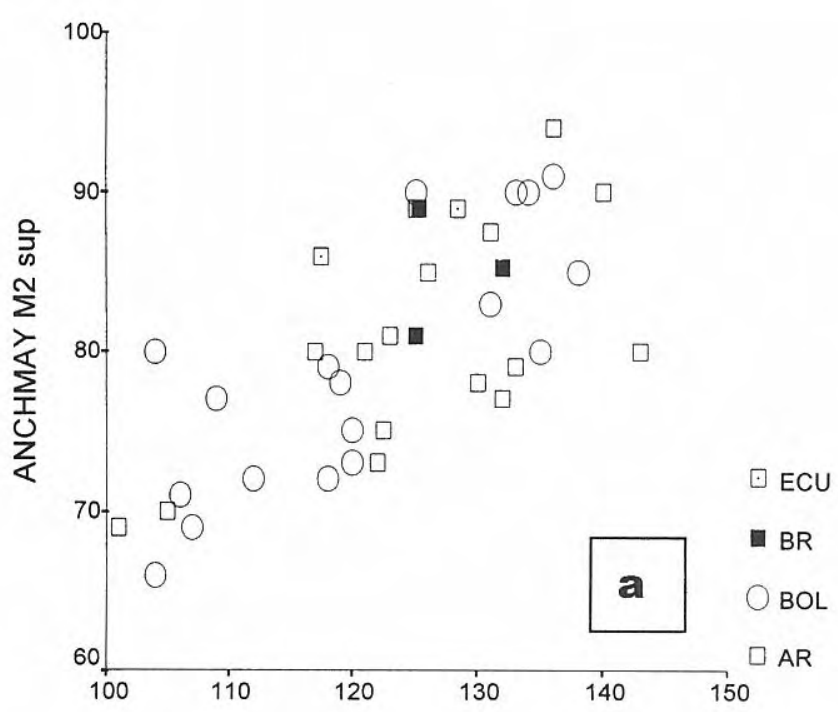

LONG M2 sup

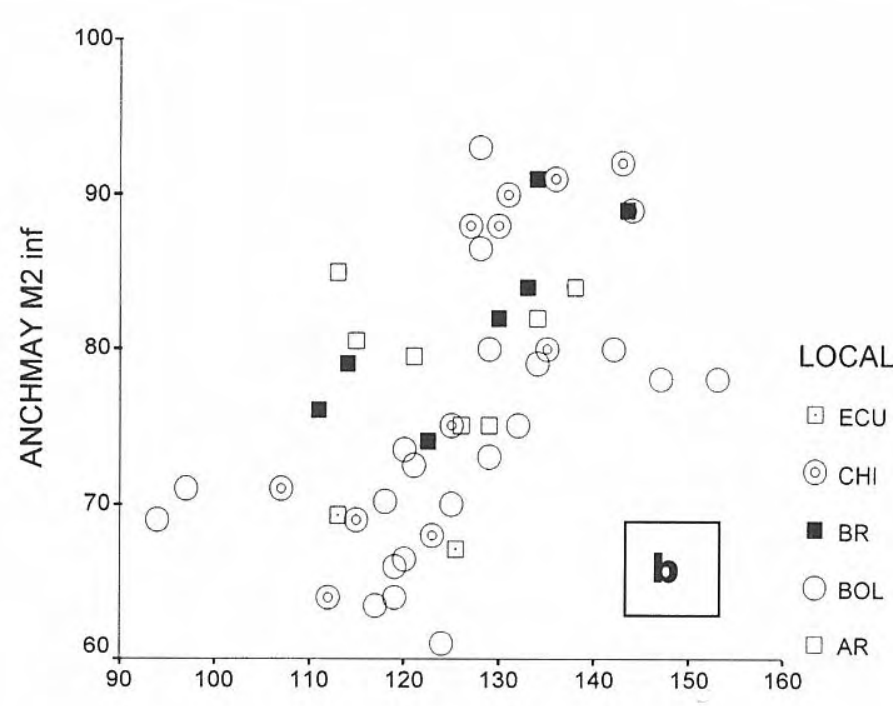

LONG M2 inf
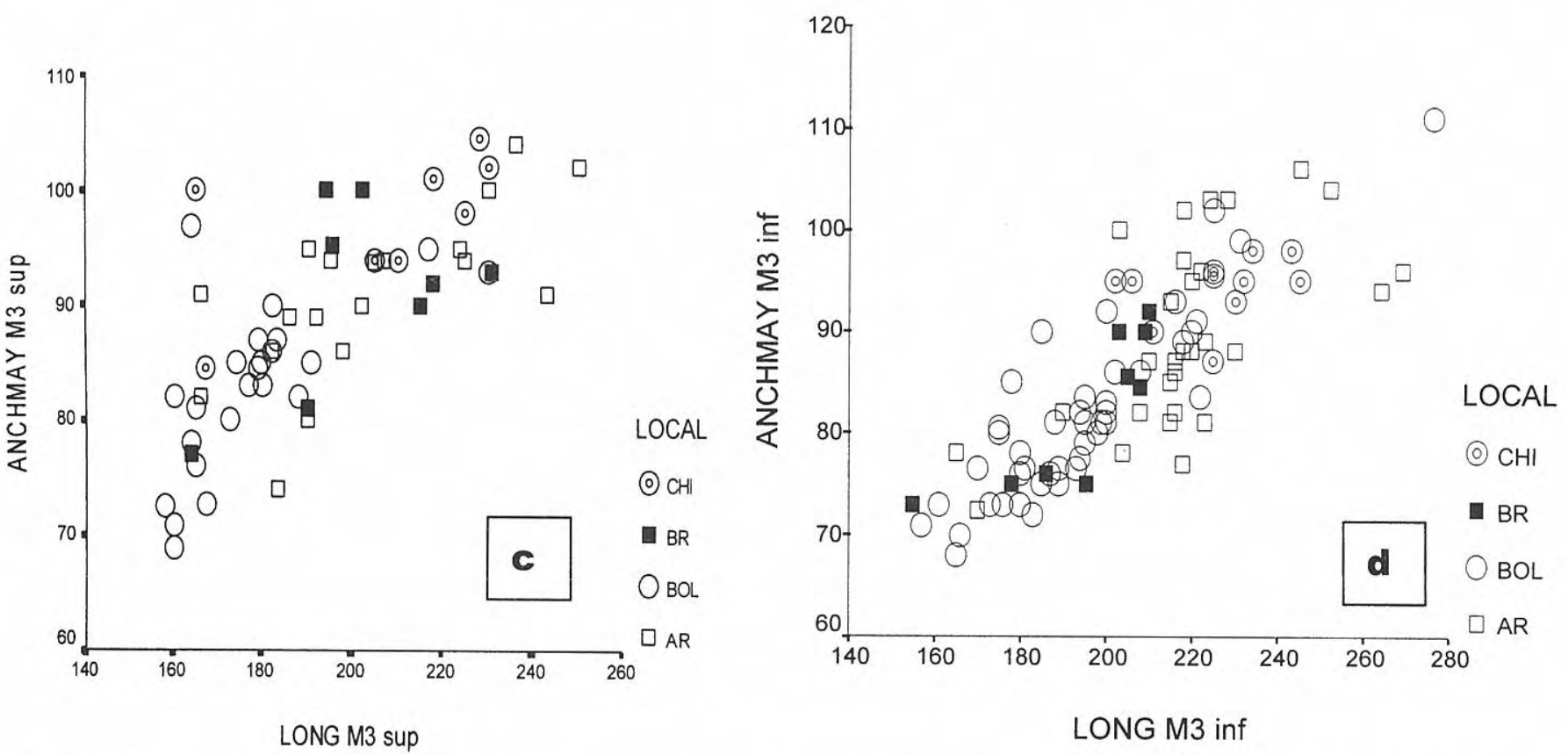

Figura 5. Diagramas bivariantes de los segundos y terceros molares superiores e inferiores, M2 y M3, del Gomphotheriidae de Brasil (BR): Stegomastodon waringi, comparados con los mismos molares procedentes de otros países de América del Sur. ECU: Ecuador; BOL: Bolivia; AR: Argentina; CHI: Chile. a: longitud por anchura de la colina mayor en M2 superiores; b: longitud por anchura de la colina mayor en M2 inferiores; c: longitud por anchura de la colina mayor en M3 superiores; d: longitud por anchura de la colina mayor en M3 inferiores.

Bivariate diagram of the lower and upper M2 and M3, of Gomphotheriidae from Brazil (BR): Stegomastodon waringi, compared with the same teeth from other South American countries. ECU: Equator; BOL: Bolivia; AR: Argentina; CHI: Chile. a: maximal length versus maximal width of upper M2; $\boldsymbol{b}$ : maximal length versus maximal width of lower M2; : maximal length versus maximal width of upper M3; d: maximal length versus maximal width of lower M3.

afloramiento. La zona de la bóveda craneana falta prácticamente entera. La zona anterior del maxilar o sínfisis presenta los alvéolos de las defensas algo rotos, con restos de defensa en el alvéolo izquierdo, similares a las del cráneo de Pains, y por tanto más bien rectas, poco divergentes. El D2 tiene dos colinas, cíngulo fuerte y una cierta anancoidía. D3 ya tiene 3 colinas y talón fuerte, y ángulo entre conos externos e internos en la $2^{\mathrm{a}}$ y $3^{\mathrm{a}}$ colina (figurado en Simpson y Paula Couto, 1957: Tabla 18). Las dimensiones siguiendo los parámetros especificados en la Figura 2 están en la Tabla 3.

En el Museo ZMK, procedente de la Cueva Escrivania, en la región de Lagoa Santa, se encuentra un cráneo juvenil que conserva parte de la cara y una pequeña defensa recta aflorando en el alvéolo derecho (figurado en Winge 1906: 


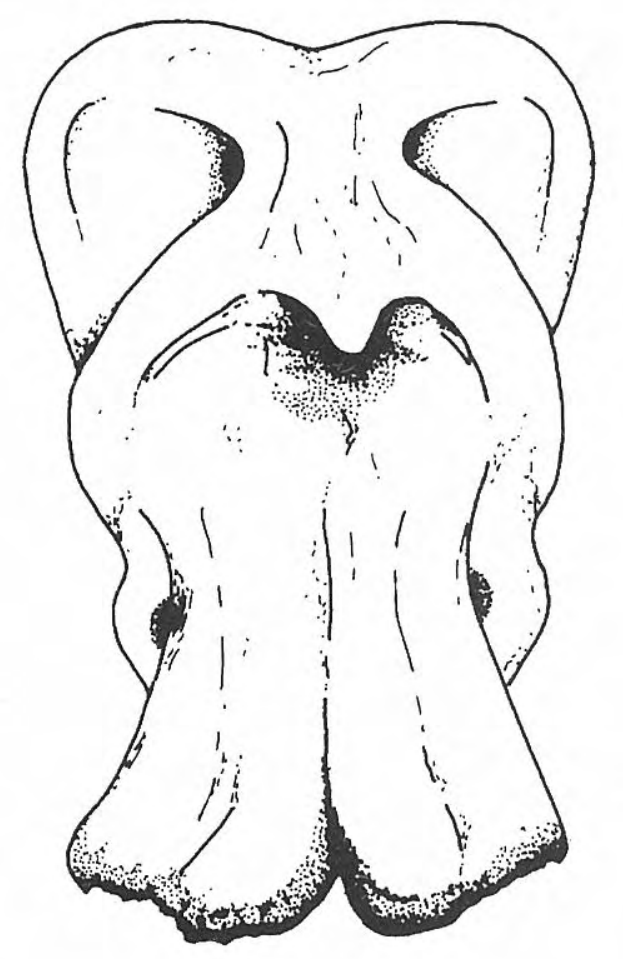

Lám. IX, y Simpson y Paula Couto, 1957: Lám. 17). En la cara se observan los orificios nasales y la frente está un poco deformada. La morfología de la cara dorsal, así como la vista ventral de este cráneo juvenil tiene el mismo diseño morfológico que el depositado en el MHN, y el observado en el cráneo de Pains, a pesar de la diferencia de edad con este último. Este cráneo conserva el D2 y el D3 izquierdo y derecho. El D2 con dos colinas y el D3 con tres colinas y talón, no presentan ni ángulo entre los conos externos e internos, ni anancoidía, pero sí división ternaria, sobre todo, en los conos externos. Dimensiones en Tabla 3.

En el MNRJ se encuentra una mandíbula completa (53-V y 54-V) que podría proceder de Pesqueira (Pernambuco), sin confirmar. Esta mandíbula es más bien baja y conserva M2 y M3 derechos e izquierdos, así como las ramas ascendentes y los cóndilos de articulación con el cráneo. Nomenclatura y dimensiones siguiendo la Figura 3. La fosa masetérica (4) está bastante al borde del proceso coronoide (1), y es grande en ambos lados. La incisura mandibular (2) también está bien marcada y muy cerca del proceso coronoide, por encima, y por debajo del cóndilo articular (3). El proceso coronoide es liso e inclinado sin hacer saliente; la incisura de la mandíbula es alargada y ascendente, terminando en el cóndilo articular (3) bien desarrollado; rama ascendente mandibular no muy ancha; fosa pterigoidea (11) alargada, profunda y oval, siguiendo la inclinación de la rama ascendente; borde mandibular (6) recurvado suave; los forámenes mentonianos (8), en la parte anterior de la mandíbula, están claramente marcados; la sínfisis mandibular (9) es algo alargada y está ligeramente curvada hacia abajo, su extremo mesial. El borde lateral de la sínfisis o cresta interalveolar forma un ángulo con la superficie oclusal de la dentición $(\alpha)$ de $36^{\circ}$. La longitud de la sínfisis en el plano sagital (10) es de $142 \mathrm{~mm}$, y su altura de 132 $\mathrm{mm}$. La longitud de la serie dentaria derecha (7) es de 380 $\mathrm{mm}$, y la izquierda $340 \mathrm{~mm}$. La altura de la mandíbula a nivel anterior de la serie molar (b), M2 en este caso, es de $166 \mathrm{~mm}$ y la altura de la misma a nivel posterior de la serie molar (c), M3 en este caso, está en torno a $170 \mathrm{~mm}$. La longitud máxima de la mandíbula en el plano sagital (a) es de $700 \mathrm{~mm}$. La distancia mínima entre el borde antero interno de los molares (e) es de $108 \mathrm{~mm}$.

El análisis morfológico de los restos dentarios aislados de Brasil, en general, indica una gran uniformidad. Todos son claramente bunodontos presentando división binaria o ternaria de los conos principales. La división ternaria se observa con más frecuencia en los conos externos en superiores e internos en inferiores. De todas maneras, en los restos dentarios de Brasil, la subdivisión de conos en

Figura 6. Esquema del cráneo de Pains (MG), objeto principal de este estudio, depositado en el MCN, a partir de la reconstrucción realizada en dicho museo: a: en vista facial; b: en vista nucal; $\mathbf{c}$ : en vista lateral izquierda.

Schematic drawing of the Pains skull (Minas Gerais), stored at the MCN, from the reconstruction make in this museum: $\boldsymbol{a}$ : in facial view; $\boldsymbol{b}$ : in nucal view; $\boldsymbol{c}$ : in left lateral view. 
coneletes es mayor que en los restos dentarios de Cuvieronius procedentes de Tarija (Bolivia) y en los dientes procedentes de distintas localidades chilenas, como apuntan recientemente Frassinetti y Alberdi (2000). Los cónulos centrales aparecen, en algunos casos, situados en ambos bordes de la línea media. Estos presentan, en general, una morfología más complicada y los dibujos en forma de tréboles de desgaste también son más complicados, encontrándose tanto en los conos externos como en los internos. La línea media suele estar bien marcada, y va desapareciendo a medida que avanza el grado de desgaste del diente. En Cuvieronius, con figuras más sencillas en general, éstas son más complicadas en el lado que incluye los cónulos centrales. Los dientes intermedios M1 y M2 (incluso los D4 que se conservan) son trilofodontos, con 3 colinas completas y talón; M2, en general, presenta un talón robusto formado por dos coneletes fuertes. En general, el cíngulo mesial es fuerte prolongándose lateralmente. En algunos se observan cónulos laterales en los bordes de los valles formando parte del cíngulo.

M3 generalmente tienen 5 colinas y un talón más o menos desarrollado en superiores e inferiores. Los dientes superiores suelen ser más cortos y más anchos que los inferiores. M3, en general, presenta cíngulo mesial fuerte, que suele prolongarse externa (en inferiores) o internamente (en superiores), llegando en algunos casos hasta el talón. También se observa en las colinas $4^{\mathrm{a}}$ y $5^{\mathrm{a}}$, algunas veces también en la $3^{a}$, e incluso sobre el talón, una ligera anancoidía, esto es, un cierto desplazamiento de los conos externos frente a los internos de una misma colina. Este carácter morfológico representa un avance frente a la oblicuidad de los conos externos con relación a los internos. Esta oblicuidad de los conos externos e internos entre ellos también se observa en la mayoría de los M3, en las colinas $3^{a}$ y $4^{a}$ y en algunos casos en la $5^{\text {a }}$, de los gonfoterios de Bolivia y Chile (Frassinetti y Alberdi, 2000), formando ángulo entre ellos. Esto también se observa en aquellos dientes de Brasil y Argentina que tienen una morfología menos complicada. En la mayoría de los dientes que ya están en uso se observa una capa de cemento importante rellenando los valles e incluso cubriendo los cónulos.

Los restos de mastodonte de Toca dos Ossos se reducen a dos M3 incompletos y numerosos fragmentos de dientes juveniles. Los fragmentos de M3 permiten observar los mismos caracteres que los procedentes de otras localidades brasileñas. Así mismo, los fragmentos de dientes juveniles confirman la presencia de dichos caracteres de gonfoterios, como son: dientes intermedios bunodontos con tres colinas, línea media marcada, cónulos centrales rellenando los valles, cíngulo mesial fuerte y lateral que a veces llega hasta el talón y paredes de las colinas en algunos casos con el esmalte crenulado (Fig. $7 \mathrm{~h}-\mathrm{j}$ ).

\section{DISCUSIÓN}

Los restos óseos del esqueleto apendicular no ofrecen diferencias morfológicas entre los restos procedentes de las distintas localidades de Brasil, ni en comparación con los de otras localidades de América del Sur. Sólo se observan diferencias de talla. Por su parte, las defensas presentan una alta variabilidad en forma y tamaño, incluso dentro de una misma localidad. La presencia o ausencia de banda de esmalte puede variar dependiendo de la edad del individuo, llegando incluso a desaparecer en los adultos. Hay consenso entre los autores sobre la alta variabilidad individual de este grupo de animales.

El análisis morfométrico de los restos dentarios indica que sus tamaños son muy variables, con un amplio rango de variabilidad, no sólo entre dientes superiores e inferiores sino también entre el mismo tipo de dientes. Incluso hemos comparado los restos dentarios de Brasil, con los de la población de Cuvieronius de Tarija (Bolivia), claramente mono-específica y muy numerosa en restos dentarios, donde se observa que ocurre lo mismo (Fig. 5). Es decir, el rango de variabilidad de los distintos tipos de dientes de Cuvieronius de Tarija también es muy alto. Esto se observa prácticamente en todas las localidades sudamericanas con restos de mastodontes fósiles (Frassinetti y Alberdi, 2000; Alberdi y Frassinetti, en elaboración).

Los análisis bivariantes de M2, utilizando la longitud con relación a la anchura mayor, indican un amplio rango de tamaños de las distintas localidades estudiadas, así como los de las poblaciones utilizadas como material de comparación. Esta variación en talla es frecuente en las especies actuales de elefantes y no debe utilizarse para fundamentar más de una especie de gonfoterio en el registro de Brasil (Tablas 1 y 2; Fig. 5). Los análisis bivariantes para los M3 (longitud por la anchura mayor de las colinas, Tablas 1 y 2; Fig. 5) indican prácticamente lo mismo. En la Figura 5 se puede observar, como en el caso de la localidad de Tarija (Bolivia) -que es la más rica en restos-, que la variabilidad de estos engloba a todas las demás.

La comparación morfológica del cráneo de Pains (Brasil) con los cráneos de Tarija de edades similares apunta grandes diferencias, principalmente en la morfología de la parte anterior del maxilar y los alvéolos de las defensas. El primero tiene los alvéolos de las defensas ligeramente divergentes y el maxilar relativamente recto anteriormente. En el caso de los cráneos de Tarija, los alvéolos de las defensas son tan divergentes que el maxilar tiene un aspecto bastante curvado en su parte anterior. En lo referente a la bóveda craneana, las diferencias son menores, observándose un ligero mayor abombamiento en el caso del cráneo de Pains. Pero si comparamos este último con los restos craneales argentinos, como el cráneo de Tapalqué (Prado et al., en prensa b) y el cráneo de la provincia de Carchi (Ecuador) descrito y figurado por Ficcarelli et al. (1995), no observamos diferencias importantes entre ellos. Las diferencias se reducen al tamaño de los restos, debido en gran parte a la edad correspondiente de los mismos y a pequeñas diferencias debidas a la variabilidad individual, de poco valor taxonómico. En nuestra opinión, la asignación del cráneo de Carchi (Ecuador) por Ficcarelli et al. (1995) a Haplomastodon chimborazi no está suficientemente justificada con relación al principio de prioridad y normativa del Código de Nomenclatura Zoológica (ICZN 2000). No obstante, desentrañar esta 

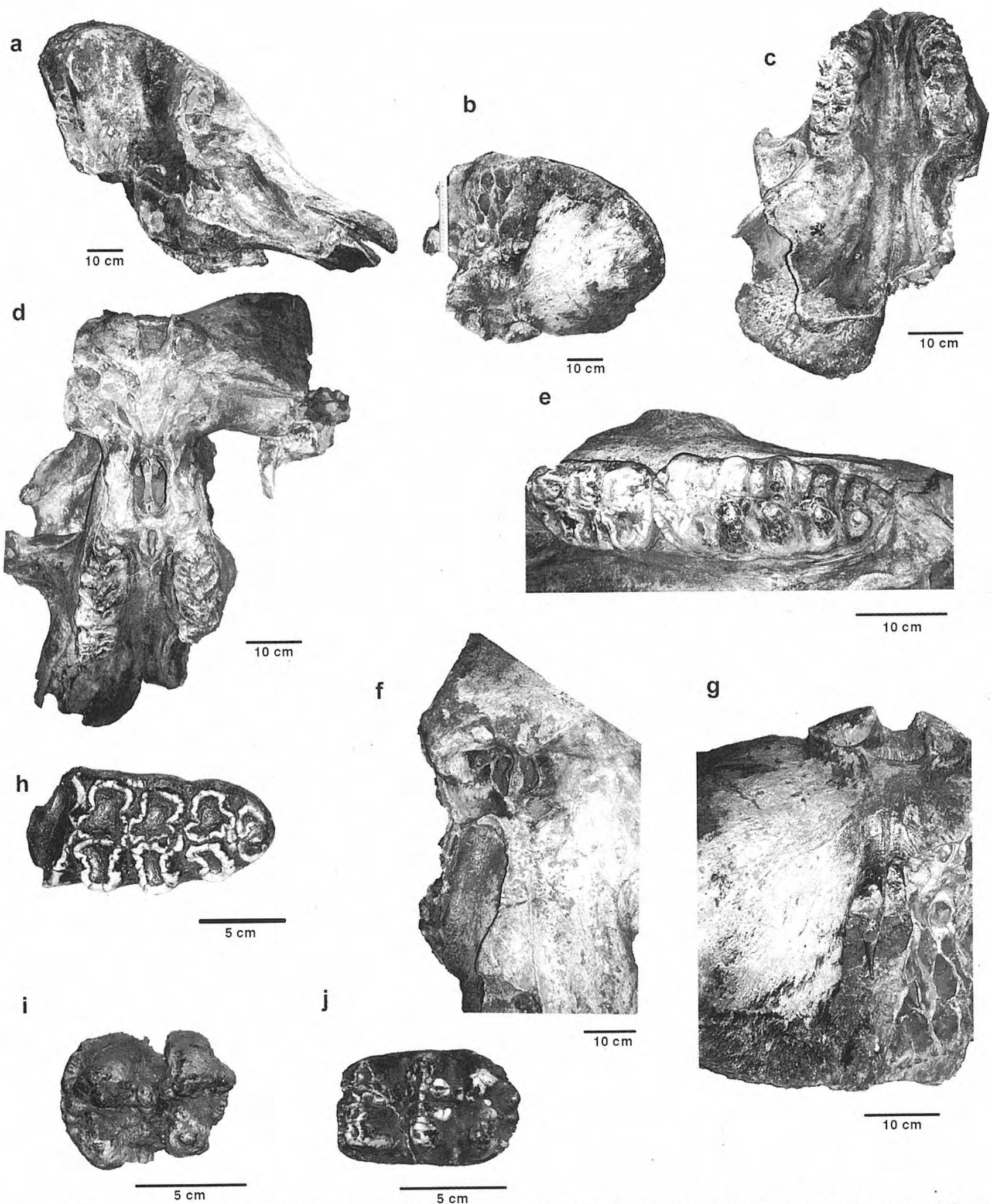

$10 \mathrm{~cm}$

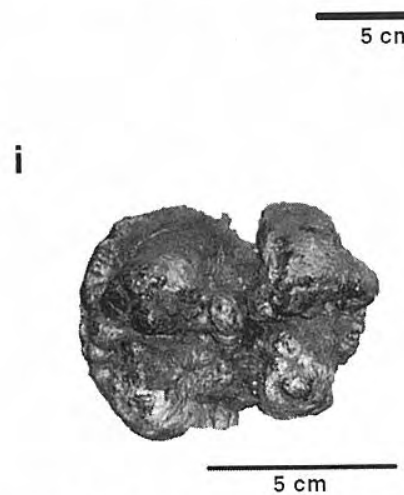

$\mathrm{cm}$ 
problemática es lo suficientemente complejo que excede los límites de este trabajo. Por otra parte, las diferencias que estos autores indican en dicho cráneo, en nuestra opinión, son más debidas a factores de crecimiento alométrico y/o caracteres debidos a dimorfismo sexual, muy acusado en este grupo. Nuestras conclusiones están de acuerdo con Simpson y Paula Couto (1957) que afirman que la especie de Ecuador es la misma que la de Brasil, y que en ninguno de los dos países se encuentran evidencias que permitan afirmar la presencia de más de una especie de gonfoterio.

\section{RADIACIÓN ADAPTATIVA DE GOMPHOTHERIIDAE EN AMÉRICA DEL SUR}

En torno a 3 Ma se registra una intensa actividad tectónica que coincide con la formación del istmo de Panamá. El mismo dio lugar a un corredor terrestre que facilitó la dispersión de plantas y animales entre ambos continentes. Este evento se conoce como el Gran Intercambio Biótico Americano (Webb, 1976, 1991). Sin embargo, este puente funcionó como un corredor de dispersión selectivo (Webb, 1978; Simpson, 1980). Los datos paleobiogeográficos indican una alternancia entre tres tipos de hábitat predominantes en este corredor durante el Plio-Pleistoceno: el bosque tropical, la sabana herbácea y la arbustiva (Webb, 1978). Durante la fase del interglaciar húmedo, los trópicos fueron dominados por bosques lluviosos, los cuales facilitaron la dispersión de la biota tropical principalmente desde América del Sur hacia Centroamérica. Mientras que, durante la fase glacial más árida, los hábitats de sabana se extendieron ampliamente a través de las latitudes tropicales y el modelo predominante fue inverso (Webb, 1991).

Simpson y Paula Couto (1957) sugirieron que todos los gonfoterios conocidos de América del Sur derivan de una radiación independiente a partir de América Central. Cuvieronius primero y luego Stegomastodon entraron en América del Sur durante la fase glacial en el Pleistoceno temprano o medio. Recientemente Campbell et al. (2000) mencionan la presencia de gonfoterios en el Mioceno tardío de la Amazonia peruana.

Ningún rasgo biológico común parece explicar por qué Mammuthus (Elephantidae) y Mammut (Mammutidae), que se registran en Florida y Honduras durante el Pleistoceno junto a gonfoterios (Rhyncotherium y Cuvieronius) y que podría esperarse que hubieran cruzado el puente terrestre panameño, no se registran en América del Sur. Las razones tal vez se deban a un comportamiento individualista. Cada uno de estos grupos presentan distintas adaptaciones a la dieta, lo cual puede traducirse como una adaptación a hábitats diferentes. Los mastodontes cigodontos (Mammut) tienen molares de corona baja con colinas separadas, que dan a la superficie oclusal un relieve desigual antes de un desgaste fuerte. Esta morfología dental sugiere que estos mastodontes eran ramoneadores (Webb et al., 1992). Por su parte, los elefantes (Mammuthus) tenían molares de corona alta con lofos de esmalte estrechamente espaciados y cubiertos con cemento. Esta morfología sugiere que eran pastadores (Davis et al., 1985). Los análisis de isótopos estables confirman estas hipótesis (MacFadden y Cerling, 1996). Los análisis de los isótopos de ${ }^{13} \mathrm{C}$ llevados a cabo sobre dientes de gonfoterios bunodontos (Stegomastodon y Cuvieronius) de América de Sur (Sánchez Chillón et al., en prensa a,b) indican que las formas del Pleistoceno medio tenían una adaptación a una dieta mixta de plantas $C_{3}$ y plantas $C_{4}$. Mientras que las formas del Pleistoceno final presentan un rango de variabilidad mayor en las dietas. Desde formas adaptadas a una dieta de plantas $\mathrm{C}_{4}$ en las llanuras costeras de Ecuador (península de Santa Elena) hasta formas estrictamente $\mathrm{C}_{3}$ en la Pampa Argentina (Sánchez Chillón et al., en prensa a,b). Esta adaptación a una dieta mixta de los primeros gonfoterios en América del Sur podría explicar por qué sólo las formas bunodontas llegaron a América del Sur cuando todos los tipos vivieron juntos en América del Norte y Centro durante el Pleistoceno.

\section{LA EXTINCIÓN DE GOMPHOTHERIIDAE EN AMÉRICA DEL SUR}

Las posibles causas que produjeron la extinción de fines del Pleistoceno son muchas, en consecuencia es difícil evaluar todos los fenómenos complejos que dieron lugar a la desaparición de una parte importante de la fauna pleistocena. Se han postulado dos tipos de teorías para explicar este fenómeno. Algunos autores atribuyen la extinción al impacto directo del hombre a través de la actividad de caza. Esta hipótesis fue principalmente sostenida por Martin (1984), quién propuso que la extinción de mamíferos grandes de América del Norte, América del Sur y Australia se relaciona con el impacto súbito de los cazadores-recolectores sobre las poblaciones naturales. Politis et al. (1995) sugieren que la presión de caza o la perturbación del hábitat afectó sobre todo a las poblaciones inmigrantes de gonfoterios y caballos pleistocenos. Entre las actividades humanas que más afectan al ecosistema está el uso del fuego para la caza. La hipótesis llamada "total overkill" se apoya en la sincronía de las extinciones y la llegada súbita de las bandas de cazadores-recolectores. Sin embargo, el registro de América del Sur no apoya esta hipótesis ya que la coexistencia entre ambos grupos se registra durante un periodo prolongado. Los gonfoterios son frecuentes en sitios paleo-indios de América del Sur. Distintas evidencias de su utilización en la alimentación provienen del sitio Monte Verde en el sur de Chile (Dillehay y Collins, 1988), Taguatagua en Chile central (Montané, 1968), Tibitó en la sabana de Bogotá, Colombia (Correal Urrego, 1981), y Taima-Taima en la Península de Coro, Venezuela (Bryan et al., 1978). Estos sitios están datados entre ca. 13.000 a 11.000 años AP. Los gonfoterios se registran en la Región Pampeana de Argentina durante este tiempo pero no en asociación con 
restos humanos. Recientemente se ha simulado por ordenador la correlación entre el aumento de las poblaciones de cazadores-recolectores y 41 especies de grandes mamíferos (Alroy, 2001). Los resultados de estas simulaciones apoyan una correlación entre la extinción de megaherbívoros y el aumento de la densidad en las poblaciones humanas.

Por otro lado, hay quienes sostienen que las extinciones se deben a cambios climáticos y ecológicos, particularmente inducidos por un estrés nutricional como consecuencia de cambios rápidos en comunidades vegetales (Graham y Lundelius, 1984; King y Saunders, 1984). Estos modelos implican que los gonfoterios eran muy especializados en su dieta y que la desaparición de su alimento específico durante la transición PleistocenoHoloceno fue el motivo del estrés nutricional. Actualmente, los elefantes africanos y asiáticos viven en hábitats bastante diversos, tienen una estrategia oportunista, y son capaces de mantenerse a base de casi cualquier mezcla de plantas (Koch et al., 1995; Bocherens et al., 1996). La mayor especialización que se nota en las poblaciones de gonfoterios del Pleistoceno superior apoyaría esta hipótesis. Recientes datos de los gonfoterios y mamut de Florida (Koch et al., 1998) muestran un patrón similar al de América del Sur que soportaría el modelo de Graham y Lundelius. Este estudio sugiere que Mammut y Mammuthus presentan hábitat y dietas distintas. Mammut muestra valores de isótopos estables que indican una dieta rica en plantas C3 y Mammuthus presenta valores más altos y más variables que indican una dieta enriquecida en pastos $\mathrm{C} 4$.

Cartelle (1999) señala que la mayoría de las grandes especies de mamíferos extintas en la zona intertropical de América del Sur tenían hábitos alimentarios preferentemente pastadores. Este autor sugiere que los cambios climáticos provocaron cambios en la zonación pluviométrica y, consecuentemente, en la composición florística. Hubo también una disminución de la sabana aumentando consecuentemente la "mata" en galería y la desertificación de la catinga, lo que contribuyó al estrés nutricional de los grandes herbívoros, la disminución de la productividad y la delimitación del territorio.

\section{CONSIDERACIONES FINALES}

Las características de los gonfoterios de América del Sur indicarían la necesidad de incluirlos todos en una única subfamilia, pero la problemática de su definición y asignación requiere de un análisis filogenético de la familia que excede los objetivos del presente trabajo. En consecuencia, nos inclinamos a dejarlos en la familia Gomphotheriidae. Nosotros reconocemos dos generos: Cuvieronius y Stegomastodon; y tres especies: Cuvieronius hyodon (Fischer), Stegomastodon waringi (Holland) y Stegomastodon platensis (Ameghino).

Se puede afirmar de acuerdo con Simpson y Paula Couto (1957) que todos los gonfoterios de Brasil corresponden a una única especie. El cráneo de Pains, así como los otros restos de mastodontes brasileños estudiados en este trabajo los asignamos a Stegomastodon waringi. Si bien los restos de Toca dos Ossos no son muy completos y muchos de ellos corresponden a individuos juveniles, creemos que los caracteres observados permiten también incluirlos en la misma especie.

En nuestra opinión, los restos de Ecuador también corresponden a una única especie: Stegomastodon waringi. En tal sentido no compartimos los criterios propuestos por Ficcarelli et al. (1995).

Desde un punto de vista general se considera que Cuvieronius habitaba los prados de altura con condiciones climáticas frías a templadas. En tanto que Stegomastodon parecería estar adaptado a llanuras con condiciones climáticas entre calurosas y húmedas a templadas.

Sánchez Chillón et al. (en prensa a,b) analizaron mediante isótopos estables la dieta de las diferentes especies de gonfoterios pleistocenos de América del Sur. Los resultados sugieren que Cuvieronius de Tarija (Bolivia) se alimentaba de una dieta mixta de plantas C3/C4. Por otro lado, Stegomastodon de Argentina tiene valores más negativos que sugieren una dieta mixta con tendencia a ramoneadora de hojas y arbustos. Las muestras analizadas de Stegomastodon waringi de la península de Santa Elena en Ecuador muestran una tendencia contraria, es decir, dieta mixta con tendencia a pastadora.

Durante el Pleistoceno en América del Sur es posible diferenciar dos vías o corredores de dispersión faunística. Estos dos corredores han condicionado la historia paleobiogeográfica de la mayoría de los mamíferos norteamericanos en América del Sur. El modelo postulado más viable para el proceso de dispersión de los gonfoterios parece indicar que el pequeño Cuvieronius utilizó el corredor de los Andes, en tanto que las formas más grandes de Stegomastodon se dispersaron a través de la ruta Oriental, y algunos se asentaron en bordes costeros (ejemplo $S$. waringi en la península de Santa Elena, Ecuador).

Cuvieronius y Stegomastodon son mastodontes bunodontos. En general, las formas bunodontas habitan áreas abiertas y de climas más secos, como la sabana o el bosque. Las preferencias de dieta entre los distintos proboscidios podrían explicar por qué sólo las formas bunodontas llegaron a América del Sur cuando ambos tipos, lofodontos y bunodontos, vivían juntos en Centro América con anterioridad a la conexión del istmo de Panamá.

\section{AGRADECIMIENTOS}

Queremos agradecer de manera especial a los conservadores/as y/o responsables de los museos e instituciones que nos han facilitado el estudio del material incluido en este trabajo, en especial a Dña. Deisi Henriques. Así mismo, los comentarios y sugerencias de las revisoras, Silvia Aramayo y Ana Mazo. La figura 6 ha sido realizada por J. Arroyo y las fotografías han sido montadas por el Servicio de Fotografía del MNCN. Este trabajo ha sido 
posible debido al Proyecto Conjunto de Investigación del Programa de Cooperación Científica con Ibero América 2000-2001 AECI (España) con Argentina. PB97-1250 DGICYT de España y proyectos de la Universidad Nacional del Centro, y el CONICET Project PIP-2000-02773, Argentina.

\section{BIBLIOGRAFÍA}

Alberdi, M.T. y Prado, J.L. 1995. Los mastodontes de América del Sur. In: Evolución biológica y climática de la Región Pampeana durante los últimos 5 millones de años. Un ensayo de correlación con el Mediterráneo occidental (Eds. M.T. Alberdi, G. Leone y E.P. Tonni). Monografías, Museo Nacional de Ciencias Naturales, CSIC, Madrid, 277-292.

Alroy, J. 2001. A Multispecies Overkill Simulation of the End-Pleistocene Megafaunal Mass Extinction. Science, 292, 1893-1896.

Bocherens, H., Koch, P.L., Mariotti, A., Geraads, D. and Jaeger, J.-J. 1996. Isotopic biogeochemistry $\left({ }^{13} \mathrm{C},{ }^{18} \mathrm{O}\right)$ of mammalian enamel from African Pleistocene hominid sites. Palaios, 11, 306-318.

Boeuf, O. 1983. Le site Villafranchien de Chilhac (HauteLoire) France. Etude Paléontologique et Biochronologique. Thèse de Doctorat d'État. Université de Paris VII, 253 pp. (inédita).

Boeuf, O. 1992. Anancus arvernensis chilhiacensis nov. subsp. (Proboscidea, Mammalia), un Mastodonte du Plio-Pléistocène de Haute-Loire, France. Geobios, 14, 179-188.

Boule, M. et Thevenin, A. 1920. Mammifères fossiles de Tarija. Paris Imp.Nat., 256 pp.

Bryan, A. 1986. Paleoamerican Prehistory as seen from South America. In: New evidence for the Pleistocene Peopling of the Americas (Ed. A. Bryan). University of Maine at Orono, 14.

Bryan, A., Casamiquela, J., Cruxent, R., Gruhn, R. and Ochsenius, C. 1978. An El Jobo Mastodon Kill at TaimaTaima, Venezuela. Science, 200, 1275-1277.

Cabrera, A. 1929. Una revisión de los Mastodontes Argentinos. Revista del Museo de La Plata, 32, 61-144.

Campbell, K.E. Jr., Frailey, C.D. and Romero, L. 2000. The late Miocene Gomphothere Amahuacatherium peruvium (Proboscidea: Gomphotheriidae) from Amazonian Peru: implications for the Great American Faunal Interchange. Instituto Geológico Minero y Metalúrgico, Boletín, 23, $1-152$.

Cartelle, C. 1999. Pleistocene Mammals of the Cerrado and Caatinga of Brazil. In: Mammals of the Neoprotropics. v.3 (Eds. J.F. Eisenberg and K.H. Redford). The University of Chicago Press, 27-46.

Casamiquela, R.M., Shoshani, J. and Dillehay, T.D. 1996. South American proboscidean: general introduction and reflections on Pleistocene extinctions. In: The Proboscidea. Evolution and Palaeoecology of Elephants and their relatives (Eds. J. Shoshany and P. Tassy). Oxford University Press, Oxford, New York, Tokyo, 316-320.
Castellanos, A. 1948. Vidal Ney - "Contribuçao ao Conhecimento da Paleontologia do Nordeste Brasileiro..." [review]. Publicaciones del Instituto de Física Geológica, República Argentina, Rosario, Crónica bibliográfica, 138-140.

Chen, G. 1999. Sinomastodon Tobien et al., 1986 (Proboscidea, Mammalia) from the Plioceno and earlymiddle Pleistoceno of China. Proceedings of the Seventh Annual Meeting of the Chinese Society of Vertebrate Paleontology, 179-187.

Correal Urrego, G. 1981. Evidencias culturales y megafauna pleistocénica en Colombia. Fundación de Investigaciones Arqueológicas Nacionales, Bogotá, 12, 1-148.

Cunha, F.L. S. de 1959. Mamíferos Fósseis do Pleistoceno do Rio Grande do Sul. I - Ungulados. Divisao de Geología e Mineralogía del Departamento Nacional da Produçao Mineral, Boletim, 202, 5-47.

Cuvier, G. 1806. Sur différentes dents du genre des mastodontes, mais d'espèces moindres que celle de l'Ohio, trouvées en plusieurs lieux des deux continens. Annales du Muséum d'Histoire Naturelle, 7, 401-420.

Davis, O.K., Mead, J.I., Martin, P.S. and Agenbroad, L.D. 1985. Riparian plants were a major component of the diet of mammoths of Southern Utah. Current Research in the Pleistocene, 2, 81-82.

Dillehay, T.D. and Collins, M.B. 1988. Early cultural evidence from Monte Verde in Chile. Nature, 332, 150152.

Dudley, J.P. 1996. Mammoths, gomphotheres, and the Great American Faunal Interchange. In: The Proboscidea. Evolution and Palaeoecology of Elephants and their relatives (Eds. J. Shoshany and P. Tassy). Oxford University Press, Oxford, New York, Tokyo, 289-295.

Ficcarelli, G., Borselli, V., Herrera, G., Moreno Espinosa, M. and Torre, D. 1995. Taxonomic remarks on the South America Mastodons referred to Haplomastodon and Cuvieronius. Geobios, 28, 745-756.

Frassinetti, D. y Alberdi, M.T. 2000. Revisión y estudio de los restos fósiles de Mastodontes de Chile (Gomphotheriidae): Cuvieronius hyodon, Pleistoceno Superior. Estudios Geológicos, 56, 197-208.

Grahan, R.W. and Lundelius, E.L. 1984. Coevolutionary disequilibrium and Pleistocene extinctions. In: Quaternary extinction: A Prehistoric Revolution (Eds. P.S. Martin and R.G. Klein). University of Arizona Press, Tucson, 223-249.

Hay, O.P. 1922. Further observations on some extinct elephants. Proceedings of Biological Society of Washington, 35, 97-102.

Hoffstetter, R. 1950. Observaciones sobre los mastodontes de Sud América y especialmente del Ecuador. Haplomastodon, subgn. nov. de Stegomastodon. Publicaciones de la Escuela Politécnica Nacional, 1, 151.

Hoffstetter, R. 1952. Les mammifères Pléistocenes de la République de l'Equateur. Mémoires de la Société Géologique de France, 66, 1-391.

Holland, W.J. 1920. Fossil mammals collected at Pedra Vermelha, Bahia, Brazil, by Gerald A. Waring. Annals Carnegie Museum, 13, 224-232. 
ICZN, 2000. Código Internacional de Nomenclatura Zoológica. Editado por el Consejo Superior de Investigaciones Científicas, $4^{\mathrm{a}}$ edición en español, 1-156.

King, J.E. and Saunders, J.J. 1984. Environmental Insularity and the Extinction of the American Mastodont. In: Quaternary extinction: A Prehistoric Revolution (Eds. P.S. Martin and R.G. Klein). University of Arizona Press, Tucson, 315-339.

Koch, P.L., Heisinger, J., Moss, C., Carlson, R.W., Fogel, M.L. and Behrensmeyer, A.K. 1995. Isotopic Tracking of Change in Diet and Habitat use in African Elephants. Science, 267, 1340-1343.

Koch, P.L., Hoppe, K.A. and Webb, S.D. 1998. The isotopic ecology of late Pleistocene mammals in North America. Part 1. Florida. Chemical Geology, 152, 119-138.

Kurten, B. and Anderson, E. 1980. Pleistocene Mammals of North America. Columbia University Press, New York, $443 \mathrm{pp}$.

Lambert, W.D. 1996. The biogeography of the gomphotheriid proboscideans of North America. In: The Proboscidea. Evolution and Palaeoecology of Elephants and their relatives (Eds. J. Shoshany and P. Tassy). Oxford University Press, Oxford, New York, Tokyo, 143-148.

Leidy, J. 1858. On new species of mastodon and elephant from Nebraska, Mastodon mirificus, Elephas imperator. Proceedings of the Academy of Natural Science of Philadelphia, 10, 10.

Lesson, R.P. 1842. Nouveau tableau du règne animal: mammifères. Paris, Arthus-Bertrand, 204 pp.

Lund, P.W. 1839. Coup-d'oeil sur les espèces éteintes de mammifères du Brésil; extrait de quelques mémoires présentés à l'Académie royale des Sciences de Copenhague. Annales Sciences Naturales, ser.2, Paris, 11, 214-234.

MacFadden, B.J. and Cerling, T.E. 1996. Mammalian herbivore communities, ancient feeding ecology, and carbon isotopes: a 10 million year sequence from the Neogene of Florida. Journal of Vertebrate Paleontology, 16, 103-115.

MacFadden, B.J., Siles, O., Zeitler, P., Johnson, N.M. and Campbell, K.E. 1983. Magnetic polarity stratigraphy of the middle Pleistocene (Ensenadan) Tarija Formation of southern Bolivia. Quaternary Research, 19, 172-187.

Martin, P.S. 1984. Prehistoric Overkill: The Globel Model. In: Quaternary extinction: A Prehistoric Revolution (Eds. P.S. Martin and R.G. Klein). University of Arizona Press, Tucson, 354-403.

Mazo, A.V. 1977. Revisión de los Mastodontes de España. Tesis doctoral, Universidad Complutense, Madrid (inédita), $450 \mathrm{pp}$.

Mones, A. y Francis, J.C. 1973. Lista de los Vertebrados fósiles del Uruguay, II. Comunicaciones Paleontológicas del Museo de Historia Natural de Montevideo, 1(4), 3997.

Montane, J. 1968. Paleoindian remains from Laguna Taguatagua Central Chile. Science, 161, 1137-1138.

Osborn, H.F. 1936. Proboscidea. A monograph of the discovery, evolution, migration and extinction of the mastodons and elephants. Vol 1-Moeritherioidea,
Deinotheiroidea, Mastodontoidea. Bulletin of the American Museum of Natural History, 1, 1-802.

Pohlig, H. 1912. Sur une vieille mandibule de "Tetracaulodon ohioticum" Blum., avec défense in situ. Bulletin de la Societé Belge de Géologie, 26, 187-193.

Politis, G.G., Prado, J.L. and Beukens, R.P. 1995. The Human Impact In Pleistocene-Holocene Extinctions. In: South America-The Pampean Case. Ancient Peoples and Landscapes (Ed. E. Johnson). Museum of Texas Tech University, Lubbock, Texas, USA, 187-205.

Prado, J.L., Alberdi, M.T., Sánchez, B. and Azanza, B. (in press a). Diversity of the Pleistocene Gomphotheres (Gomphotheriidae, Proboscidea) from South America. Deinsea.

Prado, J.L., Alberdi, M.T. and Gómez, G.N. (in press b). Late Pleistocene gomphothere (Proboscidea) remains from the Arroyo Tapalqué locality (Buenos Aires, Argentina) and their taxonomic and biogeographic implications. Neues Jahrbuch für Geologie und Paläontologie.

Proaño, J.F. 1922. La virgen del Dios Chimborazo, Tradiciones puruhaes. Riobamba, Ecuador. El Observador, 1-23.

Sánchez Chillón, B., Prado, J.L. and Alberdi, M.T. (in press a). Isotopic Evidences On the paleodiet of Pleistocene South American Gomphotheres (Gomphotheriidae, Proboscidea). Paleobiology.

Sánchez Chillón, B., Prado, J.L. and Alberdi, M.T. (in press b). Paleodiet, Ecology, and Extinction of Gomphotheres (Proboscidea) from the Pampean Region (Argentina). Coloquios de Paleontología.

Shoshani, J. 1996. Para- or monophyly of the gomphotheres and their position within Proboscidea. In: The Proboscidea. Evolution and Palaeoecology of Elephants and their relatives (Eds. J. Shoshani and P. Tassy). Oxford University Press, Oxford, New York, Tokyo, 149-177.

Simpson, G.G. 1945. The principles of classification and a classification of mammals. Bulletin of the American Museum Natural History, 85, 1-350.

Simpson, G.G. 1980. Splendid Isolation: The Curious History of South American Mammals. Yale University Press, New Haven, Connecticut, 266 pp.

Simpson, G.G. e Paula Couto, C. 1955. Os Mastodontes do Brasil. Instituto Brasileiro de Bibliografia $e$ Documentaçao, 2, 1-20.

Simpson, G.G. and Paula Couto, C. 1957. The Mastodonts of Brazil. Bulletin of the American Museum of Natural History, 112, 125-190.

Spillmann, F. 1928. Tetrabelodon nov. spec. Ayora-Spillm. El Ecuador Comercial, Quito, Ecuador 6(57), 1-70.

Spillmann, F. 1931. Die Säugetiere Ecuadors im Wandel der Zeit. Quito, Ecuador. Verlag Universidad Central, 112 pp.

Tassy, P. 1983. Les Elephantoidea Miocènes du Plateau du Potwar, Groupe de Siwalik, Pakistan. Annales de Paléontologie (Vert.-Invert.) 69: I ere Partie: Introduction, Cadre Chronologique et Geographique, Mammutidés, Amébélodontidés 99-136. II ${ }^{\mathrm{e}}$ Partie: Choerolophodontes et Gomphothères 235-297. III 
Partie: Stégodontidés, Eléphantö̈des indéterminés. Restes postcraniens. Conclusions, 317-354.

Tassy, P. 1996. How is who among the Proboscidea? In: The Proboscidea. Evolution and Palaeoecology of Elephants and their relatives (Eds. J. Shoshani and P. Tassy). Oxford University Press, Oxford, New York, Tokyo, 3948.

Tassy, P. and Shoshani, J. 1996. Historical overview of classification and phylogeny of the Proboscidea. In: The Proboscidea. Evolution and Palaeoecology of Elephants and their relatives (Eds. J. Shoshani and $\mathrm{P}$. Tassy). Oxford University Press, Oxford, New York, Tokyo, Appendix B, 352-353.

Tobien, H., Chen, G.F. and Li, Y.Q. 1986. Mastodonts (Proboscidea, Mammalia) from the late Neogene and early Pleistocene of the People's Republic of China. Part.1. Historical account: the genera Gomphotherium, Choerolophodon, Synconolophus, Amebelodon, Platybelodon, Sinomastodon. Mainzer Geowissenschaftliche Mitteilungen,15, 119-181.

Tobien, H., Chen, G.F. and Li, Y.Q. 1988. Mastodonts
(Proboscidea, Mammalia) from the late Neogene and early Pleistocene of the People's Republic of China. Part.2. The genera Tetralophodon, Anancus, Stegotetrabelodon, Zygolophodon, Mammut, Stegolophodon. Some generalities on the Chinese mastodonts. Mainzer Geowissenschaftliche Mitteilungen, 17, 95-220.

Webb, S.D. 1976. Mammalian fauna dynamics of the great American interchange. Paleobiology, 2, 220-234.

Webb, S.D. 1978. A history of savanna Vertebrates in the New World. Part II: South America and the Great Interchange. Annales Review Ecology Sistematic, 9, 393426.

Webb, S.D. 1991. Ecogeography and the Great American Interchange. Paleobiology, 17, 266-280.

Webb, S.D., Dunbar, J. and Newsom, L. 1992. Mastodon digesta from North Florida. Florida Geological Survey, Special Publication, 10, 1-59.

Winge, H. 1906. Jordfundne og nulevende Hovdyr (Ungulata) fra Lagoa Santa, Minas Geraes, Brasilien. Copenhagen, E. Museo Lundii, 3(1), 1-9.

Manuscrito recibido: 19 de septiembre, 2001 Manuscrito aceptado: 13 de marzo, 2002 